\title{
A catalogue of the vertebrate fossil record from the Guadix-Baza Basin (SE Spain)
}

\author{
Elías MALDONADO-GARRIDO ${ }^{1,2^{*}}$, Pedro PIÑERO ${ }^{1,2}$ \& Jordi AGUSTÍl, ${ }^{1,23}$
}

${ }^{1}$ IPHES, Institut Català de Paleoecologia Humana i Evolució Social; Zona Educacional 4 - Campus Sescelades URV (Edifici W3), 43007 Tarragona, Spain; eliasarte@hotmail.com; ppinero@iphes.cat

2 Àrea de Prehistòria, Universitat Rovira i Virgili (URV), Avinguda de Catalunya 35, 43002 Tarragona, Spain

${ }^{3}$ ICREA, Institució Catalana de Recerca i Estudis Avançats, Barcelona, Spain; jordi.agusti@icrea.cat

* Corresponding author

Maldonado-Garrido, E., Piñero, P. \& Agustí, J. 2017. A catalogue of the vertebrate fossil record from the Guadix-Baza Basin (SE Spain). [Catálogo del registro fósil de vertebrados de la Cuenca de Guadix-Baza (SE de España)]. Spanish Journal of Palaeontology, 32 (1), 207-236.

Manuscript received 27 October 2016

Manuscript accepted 9 April 2017

(C) Sociedad Española de Paleontología ISSN 2255-0550

\section{ABSTRACT}

In this paper, a catalogue of the vertebrate content of the Guadix-Baza Basin is presented. A total of 93 localities have been reviewed, providing more than 300 vertebrate species. These localities have been included in 11 biochronological units, ranging in age from the late Miocene (MN 13) to the Middle Pleistocene (MmQ 4), including also two MN 10 localities. The best-represented epoch in the Guadix-Baza Basin is the Pliocene (MN 14 to MN 16), with 41 sites. This is followed by the Early Pleistocene (MN 17 and MmQ1 to MmQ 3) with 30 sites. The vertebrate classes represented in the basin are Actinopterygii, Amphibia, Aves, Reptilia and Mammalia, this last one being the best represented. Among mammals, small mammals are in their turn the most abundant, particularly rodents. The families Muridae and Arvicolidae dominate the rodent assemblages, murids being the dominant family in the late Miocene and early Pliocene, and arvicolids the most abundant group in the late Pliocene and Pleistocene.

Keywords: Guadix-Baza Basin, Pliocene, Pleistocene, mammals, reptiles, amphibians.

\section{RESUMEN}

En este trabajo se presenta un catálogo de los vertebrados fósiles de la cuenca de Guadix-Baza. Se han revisado un total de 93 localidades, las cuales han proporcionado más de 300 especies de vertebrados. Las diferentes localidades han sido agrupadas en 11 unidades biocronológicas, desde el Mioceno superior (MN 13) hasta el Pleistoceno Medio (MmQ 4), incluyendo además dos localidades asignadas a la unidad MN 10. La época mejor representada es el Plioceno (MN 14 a MN 16), con un total de 41 localidades. Le sigue el Pleistoceneo Inferior (unidades MN 17 y MmQ1 a MmQ $3)$, con un total de 30 localidades. Las clases de vertebrados reconocidas en la cuenca son los Actinopterygii, Amphibia, Aves, Reptilia y Mammalia, esta última clase siendo la mejor representada en la cuenca. Entre los mamíferos, es el orden Rodentia el mejor representado. Dentro de este orden son las familias Muridae y Arvicolidae las más dominantes. Los múridos constituyen el componente mayoritario de las asociaciones del Mioceno superior y Plioceno inferior, en tanto que los arvicólidos pasan a ser la familia dominante durante el Plioceno superior y el Pleistoceno.

Palabras clave: Cuenca de Guadix-Baza, Plioceno, Pleistoceno, mamíferos, reptiles, anfibios. 


\section{INTRODUCTION}

With more than 90 fossiliferous levels, the Guadix-Baza Basin (SE Spain) contains one of the most complete Pliocene-Pleistocene continental sequences from Europe (Fig. 1). This succession includes the first evidence of hominin presence in Western Europe (Toro et al., 2013). Guadix-Baza is an intramontane Neogene and Quaternary basin of an extension of approximately $3000 \mathrm{~km}^{2}$, which is located in the Betic Chain. This basin was part of the corridors connecting the Atlantic and Mediterranean seas during part of Miocene (see general sedimentary schemes by Fernández et al., 1996a, 1996b; Soria et al., 1992, 1998). In the latest Miocene the basin was disconnected from the sea and continental successions were deposited (MinwerBarakat et al., 2009a, 2009b, 2012a, 2012b; Hüsing et al., 2010, 2012; Martín-Suárez et al., 2012). Thus, these successions provide an almost continuous continental record for the last $6 \mathrm{Ma}$. The Guadix-Baza Basin splits into two sub-basins: Guadix to the east and Baza to the west. These two sub-basins underwent several connections and disconnections as a function of both tectonics and the varying development of the alluvial and fluvial network, which are regarded as the Guadix formation (see Viseras, 1991). This formation is build up of a main basin-axis aligned deposits (i.e, east-west) known as the longitudinal system. Other supplies were derived from the basin margins to the north and south, known as the transversal systems. The alternating development of all these systems created confined lacustrine systems in diferent places of the basin (Fernández et al., 1996a, 1996b; Viseras et al., 2004, 2005). The distal lacustrine facies of the Guadix Formation are the Baza and Gorafe-Huélago formations, (Vera, 1970). Thus, when the longitudinal system was more active, lacustine systems were mainly developed in the Baza sub-basin and are those of the Baza formation.

The continental biostratigraphic record of the GuadixBaza Basin ranges from the late Miocene (late Turolian, MN 13) to the Middle Pleistocene. In addition, the basin accounts with the MN 10 locality of Cortijo de la Piedra (Sesé, 1989; Martín-Suárez et al., 2012). For biostratigraphical purposes, the 93 fossiliferous sites recognized have been grouped in biochronological units already established for the Neogene and Quaternary (Table 1). For the Neogene, we have used the MN system, established by Mein (1975; MN 13 to MN 17) while for the Quaternary we have used the MmQ system, established by Agustí et al. (1986, 1987a, MmQ 1 to MmQ 4). A total of 11 biochronological units (including MN 16a, MN 16b, MmQ 3a and MmQ 3b) have been recognized in the basin. Besides, several biozonations have been proposed for the Guadix-Baza Basin. Most of the biozones have been defined on the basis of accurate studies of micromammals from several sites (Agustí, 1986, 1990; Agustí et al., 1987a; Martín-Suárez, 1988; Ruiz Bustos, 1990; Agustí
\& Martín-Suárez, 1994; Oms et al., 2000; Gibert et al., 2007; Minwer-Barakat et al., 2012b; Agustí et al., 2015a; Piñero \& Agustí, 2015).

\section{CATALOGUE}

ACTINOPTERYGII Klein, 1885

CYPRINIFORMES Bleeker, 1859

Cyprinidae Linnaeus, 1758

Squalius Bonaparte, 1837

Squalius aff. cephalus (Linnaeus, 1758)

Sites: Barranco León-5, Fuente Nueva-3.

References: Agustí et al. (2015b), De Marfà (2007).

Squalius pyrenaicus (Günther, 1868)

Sites: Loma Quemada-1 \& 2, Cúllar de Baza-1, Barranco del Agua, Capota-1, Cueva Tapia, Huélago-4C, Huéscar-7, Meseta Bacaire.

References: Doadrio \& Casado (1989).

Squalius aff. pyrenaicus (Günther, 1868)

Sites: Barranco León-5.

References: Agustí et al. (2015b).

Luciobarbus Heckel, 1843

Luciobarbus aff. bocagei (Steindachner, 1864)

Sites: Barranco León-5, Bacochas-1, Huéscar-3, Cañada de Murcia-1, Huéscar-7, Pino Mojón.

References: Doadrio \& Casado (1989), Agustí et al. (2015b).

\section{Luciobarbus aff. sclateri (Günther, 1868)}

Sites: Barranco León-5.

References: Agustí et al. (2015b).

Cyprinidae indet.

Sites: Colorado-2, Baza-1.

References: Guerra Merchán et al. (1991), Piñero et al. (in press).

AMPHIBIA Linnaeus, 1758

ANURA Merrem, 1820

Alytidae Günther, 1859

Discoglossus Otth, 1837

Discoglossus cf. jeanneae Busack, 1986

Sites: Barranco León-5, Fuente Nueva-3.

References: Blain (2009), Blain \& Bailon (2010). 

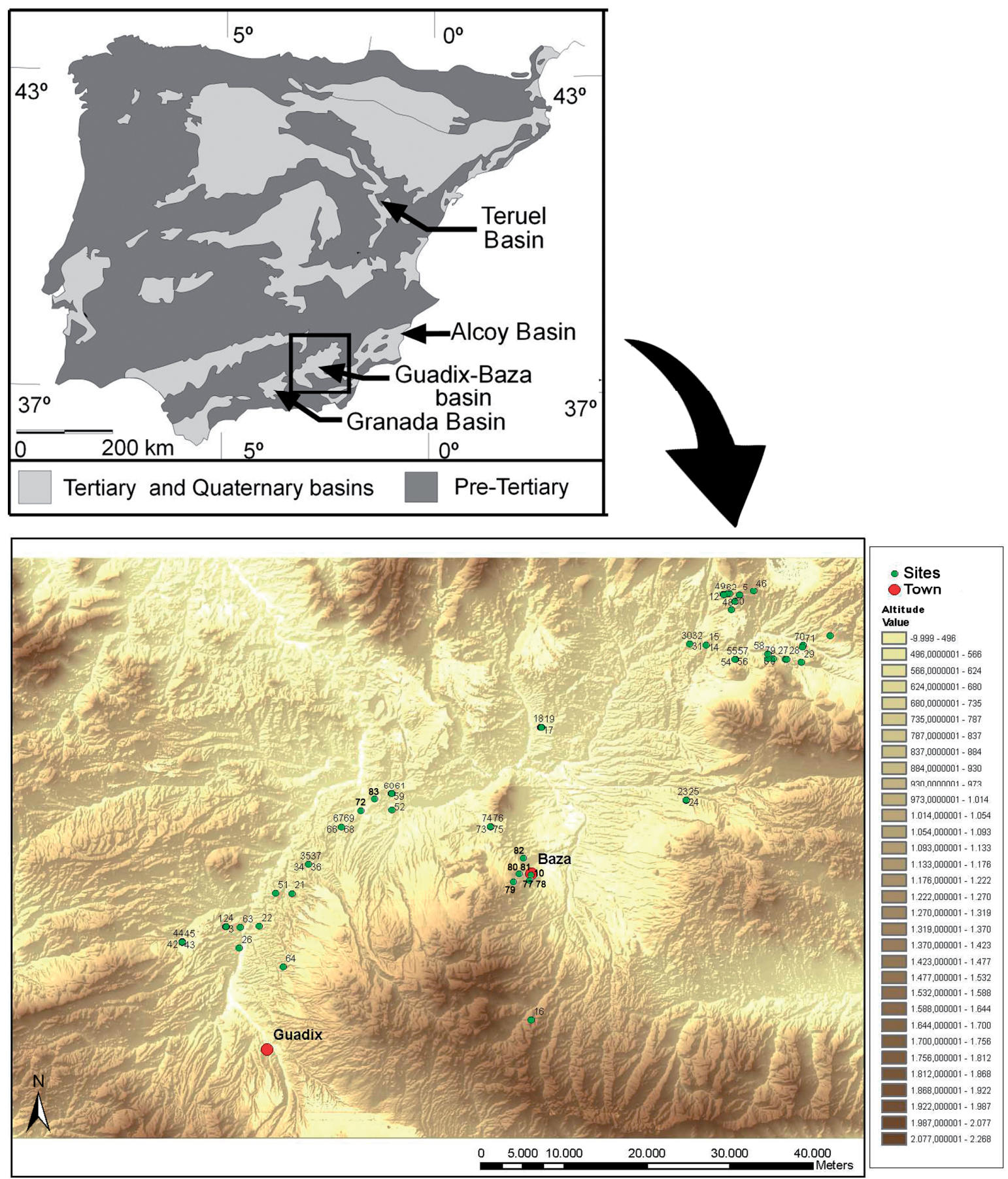

Figure 1. Geographic location of the vertebrate sites in the Guadix-Baza Basin. 1. Barranco de Cañuelas-1; 2. Barranco de Cañuelas-2; 3. Barranco de Cañuelas-3; 4. Barranco de Cañuelas-5; 5. Barranco de Quebradas-1; 6. Barranco de Paso; 7 . Barranco León; 8. Barranco León-2; 9. Barranco León-5; 10. Baza-1; 11. Canal de San Clamente-1; 12. Cañada de Murcia; 13. Cañada de Murcia-3; 14. Cañada del Castaño-1; 15. Cañada del Castaño-2; 16. Capota-1; 17. Cortes de Baza; 18. Cortes de Baza-1; 19. Cortes de Baza-6; 20. Cortijo de la Piedra; 21. Cortijo de las Sabinas; 22. Cortijo del Muro; 23. Cúllar de Baza; 24. Cúllar de Baza-1; 25. Cúllar de Baza-2; 26. Fonelas P-1; 27. Fuente Nueva-1; 28. Fuente Nueva-2; 29. Fuente Nueva-3; 30. Galera-1; 31. Galera-2; 32. Galera-3; 33. Gorafe-1; 34. Gorafe-2; 35. Gorafe-3; 36. Gorafe-4; 37. Gorafe-5; 38. Huélago; 39. Huélago-1; 40. Huélago-3; 41. Huélago-4; 42. Huélago-4C; 43. Huélago-5; 44. Huélago-6; 45. HuélagoCarretera; 46. Huéscar; 47. Huéscar-1; 48. Huéscar-3; 49. Huéscar-7; 50. Loma Quemada-1 \& 2; 51. Meseta Bacaire; 52. Negratín-1; 53. Orce-1; 54. Orce-2; 55. Orce-3; 56. Orce-4; 57. Orce-6; 58. Orce-7; 59. Pino Mojón; 60. Pino Mojón-A; 61. Pino Mojón-B; 62. Puerto Lobo; 63. Rambla del Conejo-3; 64. Solana del Zamborino; 65. Tollo de Chiclana-1; 66. Tollo de Chiclana-10 \& 10B; 67. Tollo de Chiclana-13; 68. Tollo de Chiclana-1B; 69. Tollo de Chiclana-3; 70. Venta Micena-1; 71. Venta Micena-2; 72. Yeguas; 73. Zújar-10; 74. Zújar-11; 75. Zújar-14; 76. Zújar-4; 77. Aljibe-2 \& 3; 78. Colorado-1 \& 2; 79. Cuzo-1, 2 \& 3; 80. Santa; 81. Areba; 82. Cómodo; 83. Rambla de Chimeneas-3. 
Table 1. Distribution of the vertebrate sites of the GuadixBaza Basin in the proposed Neogene and Quaternary biozones. Biozone MN 10: CP, Cortijo de la Piedra. Biozone MN 13: B-1, Bacochas-1; CO-1, Colorado-1; N-1, Negratín-1; PM-A, Pino Mojón-A; PM-B, Pino Mojón-B; SA, Salinas; RCH-3, Rambla de Chimeneas-3. Biozone MN 14: AJ-2, Aljibe-2; AJ3, Aljibe-3; BZ-1, Baza-1; CO-2, Colorado-2; CZ-1, Cuzo-1; CZ-2, Cuzo-2; CZ-3, Cuzo-3; G-1, Gorafe-1; G-4, Gorafe-4; G-A, Gorafe-A; Y, Yeguas. Biozone MN 14/MN 15: CSC-1, Canal de San Clemente-1. Biozone MN 15: BC-2, Barranco de Cañuelas-2; BC-5, Barranco de Cañuelas-5; BQ-1, Barranco de Quebradas-1; CC-1, Cañada del Castaño-1; CC-2, Cañada del Castaño-2; CÓ, Cómodo; CM, Cortijo del Muro; GA-1, Galera-1; G-2, Gorafe-2; G-3, Gorafe-3; G-5, Gorafe-5; H-3, Huéscar-3; RC-3, Rambla del Conejo-3; SN, Santa; TCH-1, Tollo de Chiclana-1; TCH-1B, Tollo de Chiclana-1B; Z-4, Zújar-4. Biozone MN 16: BC-1, Barranco de Cañuelas-1; BC-3, Barranco de Cañuelas-3; CM3, Cañada de Murcia-3; H-3, Huélago-3; H-4, Huélago-4; H-5, Huélago-5; H-6, Huélago-6; TCH-3. Tollo de Chiclana-3; TCH-13, Tollo de Chiclana-13; Z-10, Zújar-10; Z-11, Zújar-11. Biozone MN 17: COB-1, Cortes de Baza-1; COB-6, Cortes de Baza6; CT-1, Cortijo de Tapia-1; F, Fonelas P-1; FN-1, Fuente Nueva-1; G-2, Galera-2; G-3, Galera-3; H-1, Huélago-1; TCH-10, Tollo de Chiclana-10; TCH10B, Tollo de Chiclana-10B; Z-14, Zújar-14. Biozone MmQ 1: AR, Areba; BCN, Barranco de los Conejos; BP, Barranco del Paso; O-1, Orce-1; O-2, Orce-2; O-4, Orce-4. Biozone MmQ 1/MmQ 2: CS, Cortijo de las Sabinas; O-6, Orce-6; COB, Cortes de Baza. Biozone MmQ 2: CM-1, Cañada de Murcia-1; FN-2, Fuente Nueva-2; O-7, Orce-7; VM-1, Venta Micena-1; VM2, Venta Micena-2. Biozone MmQ 2/MmQ 3a: BL-2, Barranco León-2. Biozone MmQ 3a: BL-5, Barranco León-5 (Level D); FN-3, Fuente Nueva-3; O-3, Orce3. Biozone MmQ 3b: HU-1, Huéscar-1; LQ-1, Loma Quemada-1; LQ-2, Loma Quemada-2; PL, Puerto Lobo. Biozone MmQ 4: CA, Caniles; CB, Cúllar de Baza; CB-1, Cúllar de Baza-1; CB-2. Cúllar de Baza2; SZ, Solana del Zamborino.

\begin{tabular}{|c|c|c|c|}
\hline Epoch & $\begin{array}{c}\text { Mammal } \\
\text { Age }\end{array}$ & $\begin{array}{l}\text { MN and } \\
\text { MmQ units }\end{array}$ & Localities \\
\hline \multirow[b]{8}{*}{ 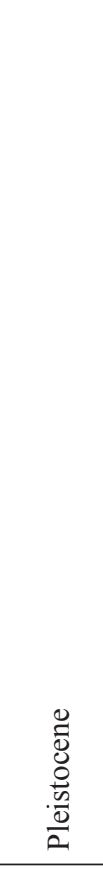 } & \multirow{7}{*}{ Biharian } & MmQ 4 & $\begin{array}{l}\text { CA, CB, CB-1, } \\
\text { CB-2, SZ. }\end{array}$ \\
\hline & & MmQ 3b & $\begin{array}{l}\text { HU-1, LQ-1, LQ- } \\
\text { 2, PL. }\end{array}$ \\
\hline & & MmQ 3a & BL-5, FN-3, O-3. \\
\hline & & $\begin{array}{l}\text { MmQ 2/MmQ } \\
3 \mathrm{a}\end{array}$ & BL-2. \\
\hline & & MmQ 2 & $\begin{array}{l}\text { CM-1, FN-2, O-7, } \\
\text { VM-1, VM-2. }\end{array}$ \\
\hline & & $\begin{array}{l}\mathrm{MmQ} \text { 1/MmQ } \\
2\end{array}$ & $\mathrm{CS}, \mathrm{O}-6, \mathrm{COB}$ \\
\hline & & MmQ 1 & $\begin{array}{l}\mathrm{AR}, \mathrm{BCN}, \mathrm{BP} \\
\mathrm{O}-1, \mathrm{O}-2, \mathrm{O}-4 .\end{array}$ \\
\hline & \multirow{2}{*}{ Villanyian } & MN 17 & $\begin{array}{l}\text { COB-1, COB- } \\
6, \text { CT-1, F, FN-1, } \\
\text { G-2, G-3, TCH-10, } \\
\text { TCH-10B, Z-14. }\end{array}$ \\
\hline \multirow[b]{4}{*}{ 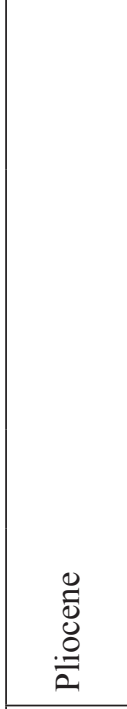 } & & MN 16 & $\begin{array}{l}\text { BC-1, BC-3, CM- } \\
3, \mathrm{H}-1, \mathrm{H}-3, \mathrm{H}-4, \\
\text { H-5, H-6, TCH-3, } \\
\text { TCH-13. }\end{array}$ \\
\hline & \multirow{3}{*}{ Ruscinian } & MN 15 & $\begin{array}{l}\text { BC-2, BC-5, BQ-1, } \\
\text { CC-1, CC-2, CÓ, } \\
\text { CM, GA-1, G-2, } \\
\text { G-3, G-5, H-3, } \\
\text { RC-3, SN, TCH-1, } \\
\text { TCH-1B, Z-4. }\end{array}$ \\
\hline & & MN 14/MN 15 & CSC-1. \\
\hline & & MN 14 & $\begin{array}{l}\text { AJ-2, AJ-3, BZ-1, } \\
\text { CO-2, CZ-1, CZ- } \\
\text { 2, CZ-3, G-1, G-4, } \\
\text { G-A, Y. }\end{array}$ \\
\hline \multirow{3}{*}{ 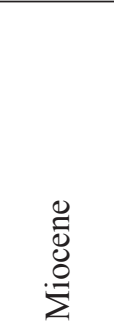 } & Turolian & MN 13 & $\begin{array}{l}\text { B-1, CO-1, N-1, } \\
\text { PM-A, PM-B, SA, } \\
\text { RCH-3. }\end{array}$ \\
\hline & & & \\
\hline & Vallesian & MN 10 & CP-1, CP-2. \\
\hline
\end{tabular}




\section{Discoglossus sp.}

Sites: Barranco de los Conejos, Galera-2, Orce-4, Cañada de Murcia-1, Loma Quemada-1 \& 2.

References: Blain (2009), Agustí et al. (2013).

Pelobatidae Bonaparte, 1850

Pelobates Wagler, 1830

\section{Pelobates cultripes Cuvier, 1829}

Sites: Barranco León-5, Fuente Nueva-3.

References: Blain (2003), Blain (2009), Blain \& Bailon (2010), Agustí et al. (2015b).

Bufonidae Gray, 1825

Bufo Laurenti, 1768

\section{Bufo bufo (Linnaeus, 1758)}

Sites: Barranco León-5.

References: Blain (2009), Blain \& Bailon (2010).

Bufo calamita (Laurenti, 1768)

Sites: Barranco León-5.

References: Blain (2003), Blain (2009), Blain \& Bailon (2010).

$$
\text { Bufo sp. }
$$

Sites: Fuente Nueva-3.

References: Blain (2003), Blain \& Bailon (2010).

Hylidae Rafinesque, 1815

Hyla Laurenti, 1768

\section{Hyla meridionalis (Boettger, 1864)}

Sites: Barranco León-5.

References: Blain (2003), Blain (2009), Blain \& Bailon (2010).

Ranidae Rafinesque, 1814

Pelophylax Fitzinger, 1843

Pelophylax cf. perezi (López-Seoane, 1885)

Sites: Galera-1, Barranco de los Conejos, Orce-4, Cañada de Murcia-1, Fuente Nueva-2, Venta Micena-1, Venta Micena-2, Barranco León-5, Fuente Nueva-3.

References: Agustí et al. (1983), Fèlix \& Montori (1987), Blain (2003), Blain (2009), Blain \& Bailon (2010), Agustí et al. (2013), Agustí et al. (2015b).

Anura indet.

Sites: Baza-1.

References: Piñero et al. (in press).
REPTILIA Laurenti, 1768

CHELONIA Latreille in Sonnini \& Latreille, 1802

Testudinidae Batsch, 1788

Mauremys Gray, 1869

Mauremys leprosa (Schweigger, 1812)

Sites: Barranco León-5.

References: Bailon (2010).

Testudo Linnaeus, 1758

\section{Testudo sp.}

Sites: Venta Micena-2, Huéscar-1, Cúllar de Baza-1, Solana de Zamborino, Cañuelas-2, Cortijo de Becerra, Barranco León-5.

References: Ruíz-Bustos (1976), Agustí et al. (1983, 1985, 1986), Martín-Penela (1988), Jiménez-Fuentes \& Martin de Jesus (1989), Blain (2009), Bailon (2010).

Testudinidae indet.

Sites: Baza-1.

References: Piñero et al. (in press).

Emydidae Rafinesque, 1815

Emys Duméril, 1806

$$
\text { Emys orbicularis (Linnaeus, 1758) }
$$

Sites: Barranco León-5.

References: Bailon (2010).

Emydidae indet.

Sites: Colorado-2.

References: Guerra Merchán et al. (1991).

SQUAMATA Oppel, 1811

Lacertidae Oppel, 1811

Acanthodactylus (Schinz, 1833)

Acanthodactylus cf. erythrurus (Schinz, 1833)

Sites: Cúllar de Baza-1.

References: Barbadillo (1989).

Podarcis Wagler, 1830

\section{Podarcis sp.}

Sites: Cúllar de Baza-1.

References: Barbadillo (1989).

Psammodromus Hallowell, 1852

Psammodromus cf. algirus (Linnaeus, 1758)

Sites: Barranco de Cañuelas-5, Cueva del Alfarero-1. 
References: Barbadillo (1989).

Timon Tschudi, 1836

Timon cf. lepidus Daudin, 1802

Sites: Barranco León-5, Fuente Nueva-3, Cúllar de Baza-1. References: Barbadillo (1989), Blain (2009), Blain \& Bailon (2010).

Lacertidae indet.

Sites: Baza-1.

References: Piñero et al. (in press).

Scincidae Gray, 1825

Chalcides Laurenti, 1768

Chalcides cf. bedriagai Boscá, 1880

Sites: Barranco de los Conejos, Fuente Nueva-3, Cúllar de Baza-1, Cueva del Alfarero-1.

References: Barbadillo (1989), Blain (2009), Blain \& Bailon (2010), Agustí et al. (2013).

Anguidae Oppel, 1811

Dopasia Gray, 1853

Dopasia sp.

Sites: Barranco León-5, Fuente Nueva-3.

References: Blain (2003, 2009), Blain \& Bailon (2010).

Anguidae indet.

Sites: Baza-1

References: Piñero et al. (in press).

Amphisbaenidae Gray, 1865

Blanus Wagler, 1830

Blanus sp.

Sites: Cúllar de Baza-1.

References: Barbadillo (1989).

Colubridae Oppel, 1811

Coronella Laurenti, 1768

Coronella cf. girondica Daudin, 1803

Sites: Barranco León-5.

References: Blain (2009), Blain \& Bailon (2010).

Coronella sp.

Sites: Barranco de los Conejos.

References: Agustí et al. (2013).

Malpolon Fitzinger, 1834
Malpolon monspessulanus (Hermann, 1809)

Sites: Barranco León-5, Fuente Nueva-3.

References: Blain (2009), Blain \& Bailon (2010).

Natrix Laurenti, 1768

$$
\text { Natrix maura Linnaeus, } 1758
$$

Sites: Galera-1, Orce-4, Barranco León-5, Fuente Nueva-3. References: Blain (2003, 2009), Blain \& Bailon (2010).

Natrix natrix (Linnaeus, 1758)

Sites: Fuente Nueva-2, Barranco León-5, Fuente Nueva-3, Cúllar de Baza-1.

References: Blain (2009), Blain \& Bailon (2010).

Rhinechis Michahelles, 1833

Rhinechis scalaris (Schinz, 1822)

Sites: Fonelas P-1, Barranco León-5, Fuente Nueva-3, Cúllar de Baza-1.

References: Laplana \& Blain (2008), Blain (2009), Blain \& Bailon (2010).

Elapidae Boie, 1827

Naja Laurenti ,1768

Naja sp.

Sites: Baza-1.

References: Piñero et al. (in press).

Viperidae Oppel, 1811

Viperidae indet.

Sites: Fonelas P-1.

References: Laplana \& Blain (2008), Blain (2009).

Scolecophidia Cope, 1864

Scolecophidia indet.

Sites: Baza-1.

References: Piñero et al. (in press).

AVES Linnaeus, 1758

ANSERIFORMES Wagler, 1831

Anatidae Vigors, 1825

Anas Linnaeus, 1758

Anas clypeata (Linnaeus, 1758)

Sites: Huéscar-1.

References: Sánchez Marco (1989).

Anas crecca (Linnaeus, 1758)

Sites: Huéscar-1.

References: Sánchez Marco (1989). 
Anas platyrhynchos Linnaeus, 1758

Sites: Huéscar-1.

References: Sánchez Marco (1989).

Anas sp.

Sites: Huéscar-1.

References: Sánchez Marco (1989).

Anas strepera (Linnaeus, 1758)

Sites: Huéscar-1.

References: Sánchez Marco (1989).

Aythya Boie, 1822

Aythya ferina (Linnaeus, 1758)

Sites: Huéscar-1.

References: Sánchez Marco (1989).

Aythya fuligula (Linnaeus, 1758)

Sites: Huéscar-1.

References: Sánchez Marco (1989).

Aythya nyroca (Güldenstädt, 1770)

Sites: Huéscar-1.

References: Sánchez Marco (1989).

$$
\text { Aythya sp. }
$$

Sites: Huéscar-1.

References: Sánchez Marco (1989).

Netta Kaup, 1829

\section{Netta rufina (Pallas, 1773)}

Sites: Huéscar-1.

References: Sánchez Marco (1989).

GALLIFORMES Temminck, 1820

Phasianidae Horsfield, 1821

Perdix Brisson, 1760

$$
\text { Perdix perdix (Linnaeus, 1758) }
$$

Sites: Huéscar-1.

References: Sánchez Marco (1989).

GRUIFORMES Bonaparte, 1854

Rallidae Vigors, 1825

Crex Linnaeus, 1758

Crex crex Linnaeus, 1758

Sites: Huéscar-1.
References: Sánchez Marco (1989).

PODICIPEDIFORMES Fürbringer, 1888

Podicipedidae Bonaparte, 1831

Tachybaptus Reichenbach, 1853

Tachybaptus ruficollis (Pallas, 1764)

Sites: Huéscar-1.

References: Sánchez Marco (1989).

STRIGIFORMES Wagler, 1830

Strigidae Vigors, 1825

Bubo Duméril, 1806

Bubo bubo (Linnaeus, 1758)

Sites: Huéscar-1.

References: Sánchez Marco (1989).

MAMMALIA Linnaeus, 1758

INSECTIVORA Bowdich, 1821

Erinaceidae Fischer, 1814

Erinaceus Linnaeus, 1758

Erinaceus cf. praeglacialis Brunner, 1934

Sites: Barranco León-5, Fuente Nueva-3.

References: Furió (2003, 2007, 2010).

Parasorex van Meyer, 1865

Parasorex ibericus (Mein \& Martín-Suárez, 1993)

Sites: Negratín-1, Rambla de Chimeneas-3.

References: Minwer-Barakat et al. (2009a, 2009b).

Erinaceidae indet.

Sites: Colorado-2, Yeguas, Baza-1, Rambla de Chimeneas-3, Barranco de Quebradas-1, Cortijo de la Piedra-2.

References: Sesé (1981), Guerra Merchán et al. (1991), Soria Mingorance \& Ruiz Bustos (1991), Minwer-Barakat et al. (2009b), Martín-Suárez et al. (2012), Piñero et al. (in press).

Soricidae Fischer, 1814

Asoriculus Kretzoi, 1959

Asoriculus gibberodon (Petényi, 1864)

Sites: Tollo de Chiclana-1, Tollo de Chiclana-1B, Yeguas, Tollo de Chiclana-13, Tollo de Chiclana-3, Venta Micena-1, Barranco León-5, Fuente Nueva-3, Orce-3, Cuzo-1.

References: Agustí et al. (1983, 1986), Martín-Suárez (1988), Guerra-Merchán \& Ruiz Bustos (1991), Furió (2003, 2010), Minwer-Barakat (2005), Minwer-Barakat et al. (2010). 
Blarinoides Sulimski, 1959

Blarinoides aliciae Minwer-Barakat, García-Alix, Martín-Suárez \& Freudenthal, 2007

Sites: Tollo de Chiclana-3.

References: Minwer-Barakat et al. (2007, 2010).

Crocidura Wagler, 1832

Crocidura kornfeldi Kormos, 1934

Sites: Galera-2, Galera-3.

References: Martín-Suárez (1988).

\section{Crocidura sp.}

Sites: Barranco León-5, Fuente Nueva-3, Orce-3, Loma Quemada-1 \& 2, Cúllar de Baza-1, Solana de Zamborino, Yeguas.

References: Ruiz-Bustos (1976), Martín-Penela (1988), Martín-Suárez (1988), Sesé (1989), Soria Mingorance \& Ruiz Bustos (1991), Furió (2003, 2007, 2010).

Myosorex Gray, 1838

\section{Myosorex meini Jammot, 1977}

Sites: Tollo de Chiclana-1B, Galera-2, Tollo de Chiclana-3, Tollo de Chiclana-10, Tollo de Chiclana-10B.

References: Furió et al. (2007), Minwer-Barakat et al. (2010).

Neomys Kaup, 1829

Neomys sp.

Sites: Cúllar de Baza-1.

References: Ruiz-Bustos (1976), Toro et al. (1984), Sesé (1989).

Paenelimnoecus Baudelot, 1972

Paenelimnoecus pannonicus (Kormos, 1934)

Sites: Tollo de Chiclana-1, Tollo de Chiclana-1B, Yeguas, Tollo de Chiclana-13, Tollo de Chiclana-3.

References: Minwer-Barakat et al. (2010).

Petenyia Kormos, 1934

Petenyia hungarica Kormos, 1934

Sites: Tollo de Chiclana-13

References: Minwer-Barakat et al. (2010).

Sorex Linneo, 1758

Sorex minutus Linnaeus, 1766
Sites: Barranco de los Conejos, Barranco León-5, Galera-2, Fuente Nueva-3, Baza-1.

References: Soria Mingorance \& Ruiz Bustos (1991), Furió (2003, 2010), Agustí et al. (2013), Piñero et al. (in press).

\section{Sorex sp.}

Sites: Barranco de los Conejos, Gorafe-2, Gorafe-3, Gorafe-5, Orce-2, Baza-1.

References: Ruiz-Bustos (1976), Agustí et al. (1987b, 2013), Martín-Penela (1988), Martín-Suárez (1988), Sesé (1989), Piñero et al. (in press).

Soricidae Indet

Sites: Negratín-1, Huéscar, Cañada del Castaño-1, Huélago-1, Tollo de Chiclana 10 \& 10b, Huéscar-1.

References: Agustí et al. (1985), Sesé (1989), MinwerBarakat et al. (2009b).

Talpidae Fischer de Waldheim, 1817

Archaeodesmana Topachevskiy \& Pashkov, 1893

Archaeodesmana brailloni (Rümke, 1985)

Sites: Gorafe-1, Gorafe-4, Tollo de Chiclana-1B.

References: Martín-Suárez (1988), Minwer-Barakat et al. (2008a).

Archaeodesmana elvirae Minwer-Barakat, García-Alix \& Freudenthal, 2008

Sites: Tollo de Chiclana-1.

References: Minwer-Barakat et al. (2008a).

Archaeodesmana dekkersi (Rümke, 1985)

Sites: Gorafe-1, Huéscar, Gorafe-4.

References: Martín-Suárez (1988).

\section{Archaeodesmana sp.}

Sites: Cañada del Castaño-1, Tollo de Chiclana-3.

References: Martín-Suárez (1988), Minwer-Barakat et al. (2008a).

Galemys Kaup, 1829

Galemys kormosi (Schreuder, 1940)

Sites: Barranco de los Conejos, Galera-2, Galera-3.

References: Martín-Suárez (1988), Agustí et al. (2013).

Galemys pyrenaicus (É. Geoffroy, 1811)

Sites: Orce-7, Venta Micena-2, Orce-3, Loma Quemada-1 $\& 2$.

References: Agustí et al. (1983, 1986), Martín-Suárez (1988). 


\section{Galemys sp.}

Sites: Venta Micena-1, Barranco León-5, Fuente Nueva-3, Orce-3.

References: Agustí et al. (1983, 1986, 1987b), MartínSuárez (1988), Furió (2003, 2010).

Talpidae indet.

Sites: Puerto Lobo, Cortijo de Tapia-1, Huélago.

References: Sesé (1989), Soria Mingorance \& Ruiz Bustos (1991).

RODENTIA Bowdich, 1821

Arvicolidae Gray, 1821

Allophaiomys Kormos, 1932

Allophaiomys aff. lavocati Laplana \& Cuenca, 2000

Sites: Barranco León-5, Fuente Nueva-3, Orce-3.

References: Agustí et al. (1990, 2010), Agustí \& Madurell (2003).

\section{Allophaiomys pliocaenicus Kormos, 1932}

Sites: Orce-7, Venta Micena-1.

References: Agustí (1991), Agustí et al. (1997).

Allophaiomys ruffoi (Pasa, 1947)

Sites: Venta Micena-1, Venta Micena-2.

References: Agustí et al. (1983, 1986, 1990), Agustí (1991).

\section{Allophaiomys sp.}

Sites: Barranco León-5, Fuente Nueva-3.

References: Agustí et al. (1987b, 1990, 2010), Agustí \& Madurell (2003).

Arvicola Lacépède, 1799

Arvicola cf. sapidus Miller, 1908

Sites: Solana de Zamborino.

References: Martín-Penela (1988), Toro et al. (1984).

Arvicola mosbachensis (Schmidtgen, 1911)

Sites: Cúllar de Baza-1.

References: Ruiz-Bustos (1976), Toro et al. (1984), Sesé (1989).

\section{Dolomys Nehring, 1898}

Dolomys adroveri Fejfar, Mein \& Moissenet, 1990

Sites: Galera-1C, Tollo de Chiclana-1.

References: Agustí (1985), Agustí et al. (1997), MinwerBarakat et al. (2004, 2008b).
Dolomys occitanus (Thaler, 1955)

Sites: Gorafe-2, Gorafe-3, Gorafe-5.

References: Martín-Suárez (1988).

Dolomys cf. occitanus (Thaler, 1955)

Sites: Galera-1C.

References: Agustí (1985), Agustí et al. (1997).

Iberomys Chaline, 1972

Iberomys brecciensis Forsyth Major, 1905

Sites: Huéscar-1, Cúllar de Baza-1, Solana de Zamborino. References: Ruiz-Bustos (1976), Toro et al. (1984), MartínPenela (1988), Sesé (1989).

Iberomys huescarensis (Ruiz Bustos, 1988)

Sites: Huéscar-1, Loma Quemada-1 \& 2, Puerto Lobo. References: Ruiz-Bustos (1988), Mazo et al. (1985), Agustí et al. (1987c).

Kislangia Kretzoi, 1954

Kislangia cappettai (Michaux, 1971)

Sites: Huélago-3, Huélago-4, Huélago-5, Huélago-6, Huélago-1, Cortijo de Tapia-1.

References: Sesé (1989).

Kislangia aff. cappettai (Michaux, 1971)

Sites: Cañada de Murcia-3.

References: Sesé (1989).

Kislangia gusii Agustí, Galobart \& MartínSuárez, 1993

Sites: Galera-2, Galera-3, Zújar-14.

References: Agustí et al. (1997, 2001).

Kislangia ischus (Esteban \& Martínez Salanova, 1987)

Sites: Zújar-11, Tollo de Chiclana-3.

References: Agustí \& Oms (1998), Agustí et al. (2001), Minwer-Barakat et al. (2004, 2008b).

Mimomys Forsyth Major, 1910

Mimomys aff. ostramosensis Janossy \& van der Meulen, 1975

Sites: Barranco de los Conejos, Barranco del Paso, Orce-2. References: Agustí (1985), Agustí et al. (1987b), Gibert et al. (1992).

Mimomys aff. pliocaenicus (Forsyth Major 1902) 
Sites: Huélago-4, Huélago-5, Huélago-1, Cortijo de Tapia-1.

References: Agustí et al. (1985, 1987b, 1987c), MartínSuárez (1988), Sesé (1989).

Mimomys cf. polonicus Kowalski, 1960

Sites: Zújar-10.

References: Agustí \& Oms (1998), Agustí et al. (2001).

Mimomys cf. reidi Hinton, 1910

Sites: Galera-1, Galera-2, Huélago-4, Huélago-1, Cortes de Baza-6, Cortijo de Tapia-1, Fuente Nueva-1, Orce-C, Alquería.

References: Agustí \& Martín-Suárez (1984), Agustí (1985), Moyá-Solá et al. (1987), Martín-Suárez (1988), Sesé (1989), Agustí et al. (1997, 2015a).

Mimomys davakosi Van de Weerd, 1979

Sites: Zújar-4.

References: Agustí et al. (2001).

Mimomys hassiacus Heller, 1936

Sites: Tollo de Chiclana-1B.

References: Minwer-Barakat et al. (2004, 2008b).

Mimomys medasensis Michaux, 1971

Sites: Galera-1, Galera-2, Galera-3, Tollo de Chiclana 10 \& 10B.

References: Martín-Suárez (1988), Sesé (1989), Agustí et al. (1997, 2015a), Minwer-Barakat et al. (2004, 2008b).

Mimomys cf. medasensis Michaux, 1971

Sites: Cortes de Baza-1, Cortes de Baza-6.

References: Martín-Suárez (1988), Sesé (1989).

Mimomys oswaldoreigi Agustí, Castillo \& Galobart, 1993

Sites: Barranco de los Conejos, Orce-2.

References: Agustí et al. (1987b, 2013).

Mimomys savini Hinton, 1910

Sites: Barranco León-5, Fuente Nueva-3, Orce-3, Huéscar-1, Loma Quemada-1 \& 2, Puerto Lobo, Areba, Caniles.

References: Agustí (1985), Agustí et al. (1987b, 2010, 2015a, 2015b), Martín-Suárez, (1988), Sesé (1989), Guerra-Merchán \& Ruiz Bustos (1991, 1992), Agustí \& Madurell (2003).

Mimomys stehlini Kormos, 1931
Sites: Huéscar-3, Tollo de Chiclana-13, Tollo de Chiclana-3, Santa.

References: Sesé (1989), Guerra-Merchán \& Ruiz Bustos (1991), Minwer-Barakat et al. (2008b).

Mimomys cf. stehlini Kormos, 1931

Sites: Barranco de Quebradas-1, Barranco de las Cañuelas-3.

References: Sesé (1989).

\section{Mimomys sp.}

Sites: Fonelas P-1, Barranco de los Conejos, Orce-3, Cómodo.

References: Agustí et al. (1987b, 2013), Laplana \& Blain (2008), Guerra-Merchán \& Ruiz Bustos (1991).

Tibericola Koenigswald, Fejfar \& Tchernov, 1992

Tibericola vandermeuleni (Agustí, 1991)

Sites: Barranco de los Conejos, Orce-2.

References: Agustí et al. (1987b, 2013).

Castoridae Hemprich, 1820

Castor Linnaeus, 1758

Castor sp.

Sites: Huélago-5, Huélago-1.

References: Sesé (1989).

Cricetidae Rochebrune, 1883

Allocricetus Schaub, 1930

\section{Allocricetus bursae}

Sites: Cúllar de Baza-1, Solana del Zamborino.

References: Sesé (1989), Martín-Penela (1988).

Apocricetus Freudenthal, Mein \& Martín-Suárez, 1998

Apocricetus alberti Freudenthal, Mein \& Martín-Suárez, 1998

Sites: Negratín-1.

References: Minwer-Barakat et al. (2009a).

Apocricetus barrierei (Mein \& Michaux, 1970)

Sites: Gorafe-1, Gorafe-A, Huéscar-3, Yeguas, Zújar-4, Botardo-C, Botardo-D, Colorado-2, Aljibe-2, Aljibe-3, Cuzo-1, Cuzo-3, Baza-1.

References: Agustí (1985), Martín-Suárez (1988), GuerraMerchán \& Ruiz Bustos (1991), Guerra-Merchán et al. (1991), Agustí \& Oms (1998), Freudenthal et al. (1998), Agustí et al. (2001), García-Alix et al. (2008), Ruiz Bustos et al. (1984), Piñero et al. (in press). 
Apocricetus cf. angustidens (Déperet , 1890)

Sites: Gorafe-2, Gorafe-3, Gorafe-5.

References: Agustí (1985), Martín-Suárez (1988).

Cricetinae indet.

Sites: Rambla de Chimeneas-3, Cortijo de la Piedra-2.

References: Minwer-Barakat et al. (2009b), Martín-Suárez et al. (2012).

Blancomys van de Weerd, Adrover, Mein \& Soria, 1977

\section{Blancomys meini Adrover, 1986}

Sites: Tollo de Chiclana-1B.

References: García-Alix et al. (2008).

Blancomys neglectus van de Weerd, Adrover, Mein \& Soria, 1977

Sites: Barranco de Quebradas-1, Huéscar-3.

References: Sesé (1989).

Hispanomys Mein \& Freudenthal, 1971

Hispanomys aff. peralensis Van de Weerd, 1976

Sites: Cortijo de la Piedra-2.

References: Martín-Suárez et al. (2012).

Rotundomys Mein, 1966

Rotundomys bressanus Mein, 1975

Sites: Cortijo de la Piedra-2.

References: Martín-Suárez et al. (2012).

Ruscinomys Depéret, 1890

Ruscinomys schaubi Villalta \& Crusafont, 1956

Sites: Salinas.

References: Soria \& Ruiz-Bustos (1992).

Ruscinomys cf. schaubi Villalta \& Crusafont, 1956

Sites: Pino Mojón-B.

References: Sesé (1989).

Ruscinomys europaeus Déperet 1890

Sites: Gorafe-5.

References: Martín-Suárez (1988).

Ruscinomys cf. europaeus Déperet 1890

Sites: Barranco de Cañuelas-5.

References: Sesé (1989).

Ruscinomys lasallei Adrover, 1969
Sites: Gorafe-A

References: Ruiz Bustos et al. (1984).

Ruscinomys cf. lasallei Adrover, 1969

Sites: Baza-1.

References: Piñero et al. (in press).

Ruscinomys sp.

Sites: Gorafe 1, Colorado 2, Negratín-1.

References: Bruijn (1974), Guerra-Merchán et al. (1991), Minwer-Barakat et al. (2009a).

Trilophomys Depéret, 1892

Trilophomys cf. castroi Adrover, 1986

Sites: Gorafe-3, Gorafe-4, Gorafe-5.

References: Martín-Suárez (1988).

Trilophomys vandeweerdi Brandy, 1979

Sites: Tollo de Chiclana-1B, Gorafe-A, Rambla del Conejo, Yeguas, Nuca-1.

References: Ruiz Bustos et al. (1984), Sesé (1989), Ruiz Bustos (1991), Minwer-Barakat (2005).

Trilophomys cf. vandeweerdi Brandy, 1979

Sites: Barranco de Cañuelas-2 \& 5, Baza-1.

References: Sesé (1989), Piñero et al. (in press).

Myocricetodon Lavocat, 1952

Myocricetodon jaegeri Benanmmi, 2001

Sites: Negratín-1.

References: Minwer-Barakat et al. (2009a), García-Alix et al. (2016).

\section{Myocricetodon sp.}

Sites: Pino Mojón-A, Pino Mojón-B, Bacochas-1.

References: Sesé (1989).

Gliridae Thomas, 1897

Eliomys Wagner, 1840

Eliomys truci Mein \& Michaux, 1970

Sites: Tollo de Chiclana-1B, Tollo de Chiclana-13, Colorado-1, Cuzo-3.

References: García-Alix et al. (2008), Guerra Merchán et al. (1991), Guerra-Merchán \& Ruiz Bustos (1991).

Eliomys cf. truci Mein \& Michaux, 1970

Sites: Cortijo de la Piedra-2, Pino Mojón-B.

References: Sesé (1989), Martín-Suárez et al. (2012). 


\section{Eliomys intermedius Friant, 1953}

Sites: Bacochas-1, Gorafe-1, Huéscar, Gorafe-4, Barranco de Quebradas-1, Cañada del Castaño-1, Cañada del Castaño-2, Gorafe-2, Gorafe-3, Gorafe-5, Rambla del Conejo-3, Tollo de Chiclana-1, Tollo de Chiclana-1B, Tollo de Chiclana-3, Cañada de Murcia-3, Galera-1, Galera-2, Galera-3, Venta Micena-1, Venta Micena-2, Barranco León-2, Orce-3, Aljibe-2, Yeguas, Baza-1.

References: Agustí et al. (1983, 1985, 1986, 1987b, 1987c, 1997), Martín-Suárez (1988), Sesé (1989), Guerra Merchán et al. (1991), Soria Mingorance \& Ruiz Bustos (1991), García-Alix et al. (2008), Piñero et al. (in press).

\section{Eliomys cf. intermedius Friant, 1953}

Sites: Barranco de Cañuelas-5

References: Sesé (1989).

\section{Eliomys quercinus Linnaeus 1766}

Sites: Huéscar-1, Cúllar de Baza, Cúllar de Baza-1, Solana de Zamborino.

References: Martín-Penela (1988), Sesé (1989).

Eliomys aff. quercinus Linnaeus 1766

Sites: Orce-2.

References: Agustí (1985), Agustí et al. (1987b).

\section{Eliomys sp.}

Sites: Barranco de los Conejos, Cortes de Baza-1, Cúllar de Baza-1, Fonelas P-1, Negratín-1.

References: Sesé (1989), Minwer-Barakat et al. (2009a), Laplana \& Blain (2008).

Muscardinus Kaup, 1829

\section{Muscardinus sp.}

Sites: Cañada del Castaño-1.

References: Martín-Suárez (1988).

Hystricidae Fischer de Waldheim, 1817

Hystrix Linnaeus, 1758

\section{Hystrix major Gervais, 1859}

Sites: Venta Micena-2.

References: Agustí et al. (1987c).

\section{Hystrix sp.}

Sites: Barranco León-5, Fuente Nueva-3.

References: Martínez-Navarro et al. (2004, 2010).

Muridae Illiger, 1811

Apodemus Kaup, 1826
Apodemus agustii Martín-Suárez, 1988

Sites: Cañada del Castaño-1.

References: Martín-Suárez (1988).

Apodemus atavus Heller, 1936

Sites: Bacochas-1, Cañada del Castaño-1, Tollo de Chiclana-1, Tollo de Chiclana-1B, Zújar-4, Galera-2, Galera-3, Tollo de Chiclana-13, Tollo de Chiclana-3, Cómodo, Santa.

References: Martín-Suárez (1988), Guerra-Merchán \& Ruiz Bustos (1991), Soria Mingorance \& Ruiz Bustos (1991), Agustí et al. (2001), Minwer-Barakat et al. (2005).

\section{Apodemus cf. atavus Heller, 1936}

Sites: Cañada de Murcia-3, Fuente Nueva-1. References: Martín-Suárez (1988).

$$
\text { Apodemus flavicollis (Melchior, 1834) }
$$

Sites: Barranco León-5, Fuente Nueva-3.

References: Agustí et al. (2010).

Apodemus cf. flavicollis (Melchior, 1834)

Sites: Solana de Zamborino.

References: Martín-Penela (1988).

Apodemus gorafensis Ruiz Bustos, Sesé, Dabrio, Pena \& Padial, 1984

Sites: Gorafe-1, Gorafe-A, Botardo-C, Colorado-1, Colorado-2, Cuzo-3, Huéscar-1, Gorafe-4, Gorafe-3, Gorafe-5, Baza-1.

References: Ruiz-Bustos et al. (1984), Martín-Suárez (1988), Sesé (1989), Guerra-Merchán \& Ruiz Bustos (1991), Guerra-Merchán et al. (1991), Piñero et al. (in press).

Apodemus gudrunae van de Weerd, 1976

Sites: Negratín-1, Huéscar, Cuzo-1, Cuzo-2, Cuzo-3.

References: Martín-Suárez (1988), Guerra-Merchán \& Ruiz Bustos (1991), Minwer-Barakat et al. (2009a).

Apodemus cf. gudrunae van de Weerd, 1976

Sites: Pino Mojón-A, Pino Mojón-B, Barranco de Cañuelas-5.

References: Sesé (1989).

Apodemus mystacinus (Danford \& Alston, 1877)

Sites: Orce-2, Orce-7, Barranco León-2, Barranco León5, Fuente Nueva-3, Venta Micena-2.

References: Agustí et al. (1987b, 1987c, 2010), MartínSuárez (1988). 
Apodemus cf. mystacinus (Danford \& Alston, 1877)

Sites: Barranco de los Conejos.

References: Agustí et al. (2013).

Apodemus aff. mystacinus (Danford \& Alston, 1877)

Sites: Barranco León-5, Venta Micena-1, Venta Micena-2. References: Agustí et al. (1983, 1986, 1987c), MartínSuárez (1988).

\section{Apodemus sylvaticus (Linnaeus, 1758)}

Sites: Orce-7, Venta Micena-1, Loma Quemada-1 \& 2, Puerto Lobo.

References: Agustí et al. (1983, 1986, 1997), Anchelergues Tarraco et al. (2015).

Apodemus aff. sylvaticus (Linnaeus, 1758)

Sites: Orce-2, Orce-7, Orce-3, Cúllar de Baza-1.

References: Ruiz-Bustos (1976), Agustí (1985), Agustí et al. (1985, 1987b), Sesé (1989).

\section{Apodemus sp.}

Sites: Huélago-4, Huélago-1, Cortijo de Tapia-1, Fonelas P-1, Barranco de los Conejos, Huéscar-1.

References: Sesé (1989), Laplana \& Blain (2008), Agustí et al. (2013).

Castillomys Michaux, 1969

Castillomys gracilis van de Weerd, 1976

Sites: Gorafe-1, Huéscar, Gorafe-4, Barranco de Quebradas-1, Huéscar-3, Baza-1.

References: Martín-Suárez (1988), Sesé (1989), Piñero et al. (in press).

Castillomys cf. gracilis van de Weerd, 1976

Sites: Barranco de Cañuelas-2, Cortijo del Muro

References: Sesé (1989).

\section{Castillomys crusafonti}

Sites: Cañada del Castaño-1, Cañada del Castaño-2, Gorafe-3, Tollo de Chiclana-1, Tollo de Chiclana-1B, Tollo de Chiclana-3, Tollo de Chiclana-13, Cañada de Murcia-3, Huélago-1, Cortes de Baza-1, Fonelas P-1, Fuente Nueva-1, Orce-2, Orce-7, Venta Micena-1, Venta Micena-2, Barranco León-2, Orce-3, Huéscar-1, Huéscar-3, Loma Quemada-1, Loma Quemada-2, Puerto Lobo, Cúllar de Baza, Gorafe-2, Gorafe-5, Galera-2, Galera-3, Huélago-4, Huélago-5, Cortijo de Tapia-1, Galera-1, Aljibe-3, Yeguas, Cómodo.

References: Agustí et al. (1983, 1985, 1986, 1987b, 1987c), Martín-Suárez (1988), Sesé (1989), Guerra-
Merchán \& Ruiz Bustos (1991), Guerra Merchán et al. (1991), Soria Mingorance \& Ruiz Bustos (1991), MinwerBarakat et al. (2005).

Castillomys rivas Martín-Suárez \& Mein, 1991

Sites: Galera-2, Tollo de Chiclana $10 \& 10 b$, Barranco de los Conejos, Barranco León-5, Fuente Nueva-3, Loma Quemada-1.

References: Minwer-Barakat et al. (2005), Agustí et al. (2010, 2013), Anchelergues Tarraco et al. (2015).

Huerzelerimys Mein, Martín-Suárez \& Agustí, 1993

Huerzelerimys minor Mein, Martín-Suárez \& Agustí, 1993

Sites: Cortijo de la Piedra-1, Cortijo de la Piedra-2.

References: Martín-Suárez et al. (2012).

Micromys Dehne, 1841

Micromys caesaris Minwer-Barakat, García-Alix, Martín-Suárez \& Freudenthal, 2008

Sites: Tollo de Chiclana-13.

References: Minwer-Barakat et al. (2008c).

Micromys minutus (Pallas, 1771)

Sites: Loma Quemada-1 \& 2.

References: Martín-Suárez (1988), Anchelergues Tarraco et al. (2015).

\section{Micromys sp.}

Sites: Tollo de Chiclana 10 \& 10B, Aljibe-3, Santa. References: Guerra Merchán et al. (1991), Soria Mingorance \& Ruiz Bustos (1991), Minwer-Barakat (2005).

Occitanomys Michaux, 1969

Occitanomys adroveri (Thaler, 1966)

Sites: Gorafe-1, Huéscar, Gorafe-4, Salinas. References: Martín-Suárez (1988), Soria \& Ruiz-Bustos (1992).

Occitanomys cf. adroveri Thaler, 1966

Sites: Pino Mojón-B.

References: Sesé (1989).

Occitanomys alcalai Adrover, Mein \& Moissenet, 1988

Sites: Negratín-1, Rambla de Chimeneas-3.

References: Minwer-Barakat et al. (2009a, 2009b). 
Occitanomys brailloni Michaux, 1969

Sites: Cañada del Castaño-1, Cañada del Castaño-2, Galera-1, Gorafe-2, Gorafe-3, Gorafe-5, Tollo de Chiclana1B, Aljibe-3.

References: Martín-Suárez (1988), Guerra Merchán et al. (1991), Agustí et al. (1997), Minwer-Barakat et al. (2005).

Occitanomys cf. brailloni Michaux, 1969

Sites: Gorafe-A, Cuzo-1, Cuzo-3, Santa, Cómodo, Colorado-2, Aljibe-3, Baza-1, Yeguas.

References: Ruiz-Bustos et al. (1984), Guerra Merchán et al. (1991), Guerra Merchán \& Ruiz Bustos (1991), Soria Mingorance \& Ruiz Bustos (1991), Piñero et al. (in press).

\section{Occitanomys hispanicus Michaux, 1971}

Sites: Cortijo de la Piedra-2.

References: Martín-Suárez et al. (2012).

\section{Occitanomys sp.}

Sites: Bacochas-1.

References: Sesé (1989).

Paraethomys Petter, 1968

Paraethomys aff. abaigari Adrover, Mein \& Moissenet, 1988

Sites: Baza-1.

References: Piñero et al. (in press).

Paraethomys jaegeri Montenat \& Bruijn, 1976

Sites: Cañada del Castaño-1, Gorafe-2, Gorafe-3, Gorafe-5, Aljibe-2, Aljibe-3.

References: Agustí (1985), Martín-Suárez (1988), Guerra Merchán et al. (1991).

\section{Paraethomys meini (Michaux, 1969)}

Sites: Negratín-1, Rambla de Chimeneas-3, Gorafe-1, Gorafe-4, Botardo-2, Botardo-3, Botardo-C, Huéscar, Tollo de Chiclana-1, Baza-1.

References: Martín-Suárez (1988), Sesé (1989), MinwerBarakat et al. (2005, 2009a, 2009b), Piñero et al. (in press).

Paraethomys cf. meini (Michaux, 1969)

Sites: Cuzo-1, Cuzo-3, Santa, Colorado-1, Colorado-2, Aljibe-2, Aljibe-3, Gorafe-A, Yeguas.

References: Ruiz Bustos et al. (1984), Guerra Merchán et al. (1991), Guerra Merchán \& Ruiz Bustos (1991), Soria Mingorance \& Ruiz Bustos (1991).

Paraethomys aff. meini (Michaux, 1969)
References: Sesé (1989).

Rhagapodemus Kretzoi, 1959

Rhagapodemus frequens Kretzoi, 1959

Sites: Tollo de Chiclana-1B.

References: Minwer-Barakat et al. (2005).

Stephanomys Schaub, 1938

Stephanomys cordii Ruiz Bustos, 1986

Sites: Gorafe-A, Yeguas, Gorafe-1, Huéscar, Gorafe-4, Aljibe-3, Botardo-C, Botardo-2, Botardo-3, Baza-1.

References: Ruiz Bustos et al. (1984), Martín-Suárez (1988), Guerra-Merchán et al. (1991), Minwer-Barakat (2005), Piñero et al. (in press).

\section{Stephanomys balcellsi Gmelig Meyling \&} Michaux, 1973

Sites: Tollo de Chiclana $10 \& 10 \mathrm{~B}$.

References: Minwer-Barakat et al. (2005).

Stephanomys cf. balcellsi Gmelig Meyling \& Michaux, 1973

Sites: Huélago-4.

References: Sesé (1989).

Stephanomys cf. margaritae Adrover, 1986

Sites: Gorafe-4, Gorafe-2, Gorafe-3, Gorafe-5.

References: Agustí (1985), Martín-Suárez (1988).

Stephanomys thaleri López Martínez, Michaux \& Hutterer, 1998

Sites: Barranco de Cañuelas-5, Barranco de Quebradas-1, Cortijo del Muro, Huéscar-3, Barranco de las Cañuelas-3, Tollo de Chiclana-13, Tollo de Chiclana-3.

References: Sesé (1989), Minwer-Barakat et al. (2005).

Stephanomys cf. thaleri López Martínez, Michaux \& Hutterer, 1998

Sites: Cañada del Castaño-1, Cañada del Castaño-2, Cañada de Murcia-3, Galera-2, Galera-3.

References: Martín-Suárez (1988).

Stephanomys aff. thaleri López Martínez, Michaux \& Hutterer, 1998

Sites: Rambla del Conejo-3.

References: Sesé (1989).

Stephanomys donnezani (Depéret, 1890)

Sites: Bacochas-1. 
Sites: Galera-1, Tollo de Chiclana-1B, Tollo de Chiclana1B, Cómodo, Santa.

References: Guerra-Merchán \& Ruiz Bustos (1991), Agustí et al. (1997), Minwer-Barakat et al. (2005).

Stephanomys cf. donnezani (Depéret, 1890)

Sites: Zújar-4.

References: Agustí \& Oms (1998), Agustí et al. (2001).

Stephanomys dubari Aguilar, Michaux, Bachelet, Calvet \& Faillat, 1991

Sites: Negratín-1, Rambla de Chimeneas-3.

References: Minwer-Barakat et al. (2009a, 2009b).

Stephanomys minor Gmelig Meyling \& Michaux, 1973

Sites: Tollo de Chiclana-13, Tollo de Chiclana-3.

References: Minwer-Barakat et al. (2005).

Stephanomys ramblensis Van de Weerd, 1976

Sites: Pino Mojón-A, Pino Mojón-B.

References: Sesé (1989).

Stephanomys aff. ramblensis Van de Weerd, 1976

Sites: Colorado-1.

References: Guerra-Merchán et al. (1991).

Stephanomys sp.

Sites: Tollo de Chiclana-1, Fonelas P-1.

References: Minwer-Barakat (2005), Laplana \& Blain (2008).

Gerbillidae Alston, 1876

Debruijnimys Castillo \& Agustí, 1996

Debruijnimys almenarensis (Agustí, 1990)

Sites: Negratín-1.

References: Minwer-Barakat et al. (2009a), García-Alix et al. (2016).

Debruijnimys julii Castillo \& Agustí, 1996

Sites: Baza-1, Bacochas-1.

References: Sesé (1989), Castillo \& Agustí (1996), Piñero et al. (in press).

\section{Debruijnimys sp.}

Sites: Botardo-C, Gorafe-1.

References: Bruijn (1974), Martín-Suárez (1988).

Sciuridae Fischer von Waldheim, 1817

Atlantoxerus Forsyth Major, 1893
Atlantoxerus adroveri (Bruijn \& Mein, 1968)

Sites: Bacochas-1, Gorafe-1, Gorafe-4.

References: Martín-Suárez (1988), Sesé (1989).

Atlantoxerus cf. adroveri (Bruijn \& Mein, 1968)

Sites: Cuzo-1.

References: Guerra-Merchán \& Ruiz Bustos (1991).

\section{Atlantoxerus margaritae Adrover, Mein \&} Moissenet, 1993

Sites: Gorafe-1.

References: García-Alix et al. (2007).

Atlantoxerus sp.

Sites: Negratín-1.

References: Minwer-Barakat et al. (2009a).

Heteroxerus Stehlin \& Schaub, 1951

\section{Heteroxerus sp.}

Sites: Cortijo de la Piedra-2.

References: Sesé (1989), Martín-Suárez et al. (2012).

Pliopetaurista Kretzoi 1962

Pliopetaurista cf. pliocaenica (Depéret, 1897)

Sites: Tollo de Chiclana-1B.

References: García-Alix et al. (2007).

Pteromyinae indet. Brandt, 1855

Sites: Cortijo de la Piedra-2.

References: Martín-Suárez et al. (2012).

LAGOMORPHA Brandt, 1855

Leporidae Fischer, 1817

Alilepus Dice, 1931

Alilepus turolensis López Martínez, 1977

Sites: Huéscar.

References: Martín-Suárez (1988).

Lepus Linnaeus, 1758

Lepus sp.

Sites: Cúllar de Baza-1, Solana de Zamborino.

References: Ruíz-Bustos (1976), Martín-Penela (1988).

Oryctolagus Lilljebord, 1874

Oryctolagus cf. cuniculus (Linnaeus, 1758)

Sites: Solana de Zamborino. 
References: Martín-Penela (1988).

Oryctolagus cf. lacosti (Pomel, 1853)

Sites: Venta Micena-1, Venta Micena-2, Barranco León-5. References: Agustí et al. (1987c, 2010), Martín-Suárez (1988), De Marfà (2007).

\section{cf. Oryctolagus sp.}

Sites: Huélago-3, Huélago-4.

References: Sesé (1989).

\section{Oryctolagus sp.}

Sites: Barranco de los Conejos, Huélago-1, Fonelas P-1, Barranco León-5, Fuente Nueva-3, Huéscar-1, Areba, Zújar-11.

References: Sesé (1989), Guerra-Merchán \& Ruiz Bustos (1991), Soria Mingorance \& Ruiz Bustos (1991), Laplana \& Blain (2008), De Marfà (2009), Agustí et al. (2013).

Trischizolagus Radulesco \& Samson, 1967

\section{Trischizolagus maritsae Bruijn, Dawson \&} Mein, 1970

Sites: Baza-1.

References: Piñero et al. (in press).

Trischizolagus aff. maritsae Bruijn, Dawson \& Mein, 1970

Sites: Barranco de Quebradas-1, Huéscar-3.

References: Sesé (1989).

Ochotonidae Thomas, 1897

Prolagus Pomel, 1853

\section{Prolagus calpensis Major, 1905}

Sites: Barranco de los Conejos, Galera-1, Galera-2, Venta Micena-1.

References: Agustí et al. (1983, 1986, 2013), MartínSuárez (1988), De Marfà (2009).

\section{Prolagus cf. calpensis Major, 1905}

Sites: Barranco de Cañuelas-2, Barranco de Cañuelas-5, Barranco de las Cañuelas-3, Fonelas P-1.

References: Sesé (1989), Laplana \& Blain (2008).

Prolagus ibericus López Martínez, 1975

Sites: Gorafe-2, Gorafe-3, Gorafe-5.

References: Martín-Suárez (1988).

Prolagus major López Martínez, 1977

Sites: Huéscar.
References: Martín-Suárez (1988).

Prolagus michauxi López-Martínez \& Thaler, 1975

Sites: Bacochas-1, Gorafe-1, Gorafe-A, Huéscar, Gorafe-4, Cañada del Castaño-1, Gorafe-2, Gorafe-3, Gorafe-5, Huéscar-3, Baza-1, Zújar-11.

References: Martín-Suárez (1988), Ruiz Bustos et al. (1984), Sesé (1989), De Marfà (2009), Piñero et al. (in press).

Prolagus cf. michauxi López-Martínez \& Thaler, 1975

Sites: Colorado-1, Colorado-2, Aljibe-2, Aljibe-3, Cuzo1, Cuzo-3.

References: Guerra Merchán et al. (1991), Guerra-Merchán \& Ruiz Bustos (1991)

\section{Prolagus sp.}

Sites: Cortijo de la Piedra, Pino Mojón-A, Pino Mojón-B, Cortijo del Muro, Rambla del Conejo-3, Huélago-4, Huélago-5, Huélago-6, Huélago-1, Cortijo de Tapia-1, Barranco León-5, Fuente Nueva-3, Yeguas, Cómodo. References: Sesé (1989), Guerra-Merchán \& Ruiz Bustos (1991), Soria Mingorance \& Ruiz Bustos (1991), De Marfà (2009), Agustí et al. (2010).

PRIMATES Linnaeus, 1758

Hominidae Gray, 1825

Homo Linnaeus, 1758

Homo sp.

Sites: Barranco León-5.

References: Toro et al. (2013).

Cercopithecidae Gray, 1821

Macaca Lacepede, 1799

Macaca sylvanus Linnaeus, 1758

Sites: Solana de Zamborino.

References: Martín-Penela (1983, 1988).

CARNIVORA Bowdich, 1821

Canidae Fischer de Waldheim, 1817

Canis Linnaeus, 1758

Canis occitanus Garrido \& Arribas, 2008a

Sites: Fonelas P-1.

References: Garrido \& Arribas (2008a).

Canis cf. lupus Linnaeus 1758

Sites: Solana de Zamborino.

References: Martín-Penela (1988). 
Canis etruscus Forsyth-Major, 1877

Sites: Fonelas P-1, Venta Micena-2, Huéscar-1, Cúllar de Baza-1.

References: Ruíz-Bustos (1976), Agustí et al. (1983, 1985, 1986), Toro et al. (1984), Pons-Moyà (1987), MartínezNavarro (1991), Alcalá \& Morales (1989), Garrido (2006, 2008a).

Canis cf. falconeri Forsyth-Major, 1877

Sites: Fonelas P-1.

References: Garrido (2008a).

Canis mosbachensis Linnaeus, 1758

Sites: Barranco León-5, Fuente Nueva-3, Venta Micena-1, Venta Micena-2.

References: Agustí et al. (1983, 1986), Pons-Moyà (1987), Martínez-Navarro (1991), Martínez-Navarro et al. (2003, 2004, 2010).

Lycaon Brookes, 1827

Lycaon lycaonoides Kretzoi, 1938

Sites: Barranco León-5, Fonelas P-1, Fuente Nueva-3, Venta Micena-2.

References: Agustí et al. (1983, 1986), Pons-Moyà (1987), Martínez-Navarro (1991), Garrido (2006), MartínezNavarro et al. (2010).

Vulpes Frisch, 1775

Vulpes alopecoides (Forsyth-Major, 1877)

Sites: Fonelas P-1.

References: Garrido (2006, 2008a).

Vulpes praeglaciaris Kormos, 1932

Sites: Cúllar de Baza-1, Venta Micena-1.

References: Ruíz-Bustos (1976), Agustí et al. (1985), Pons-Moyà (1987).

Vulpes cf. praeglacialis Kormos, 1932

Sites: Barranco León-5, Fuente Nueva-3.

References: Martínez-Navarro et al. (2003, 2004, 2010).

Felidae Fischer de Waldheim, 1817

Acinonyx Brookes, 1828

Acinonyx pardinensis (Croizet \& Jobert, 1828)

Sites: Fonelas P-1.

References: Garrido (2006).

Felis Linnaeus, 1758
Felis silvestris Schreber, 1775

Sites: Solana de Zamborino.

References: Martín-Penela (1988).

Homotherium Fabrini, 1890

Homotherium latidens (Owen 1846)

Sites: Fonelas P-1, Venta Micena-1, Venta Micena-2.

References: Agustí et al. (1983, 1985, 1986), Pons-Moyà (1987), Martínez-Navarro (1991), Garrido (2006), Arribas et al. (2008).

\section{Homotherium sp.}

Sites: Barranco León-5, Huéscar-1.

References: Alcalá \& Morales (1989), Martínez-Navarro et al. $(2003,2004,2010)$.

Lynx Kerr, 1792

Lynx cf. pardina (Temminck, 1827)

Sites: Solana de Zamborino.

References: Martín-Penela (1988).

Lynx issiodorensis (Croizet \& Jobert, 1828)

Sites: Fonelas P-1.

References: Garrido (2006).

Lynx sp.

Sites: Fuente Nueva-3, Venta Micena-2, Solana de Zamborino.

References: Agustí et al. (1983, 1986), Pons-Moyà (1987), Martín-Penela (1988), Martínez-Navarro (1991), MartínezNavarro et al. (2010).

Megantereon (Croizet \& Jobert, 1828)

Megantereon whitei (Broom, 1937)

Sites: Venta Micena-2.

References: Martínez-Navarro (1991).

Megantereon cultridens roderici Arribas \& Garrido, 2008

Sites: Fonelas P-1.

References: Garrido (2006), Arribas et al. (2008).

Megantereon cultridens adroveri Pons-Moyà, 1987.

Sites: Venta Micena-2.

References: Agustí et al. (1983, 1986), Pons-Moyà (1987).

Panthera Oken, 1816 
Panthera cf. gombaszogensis Kretzoi 1938

Sites: Venta Micena-2.

References: Agustí et al. (1983, 1985, 1986), Pons-Moyà (1987), Martínez-Navarro (1991).

\section{Panthera gombaszoegensis Kretzoi 1938}

Sites: Huéscar-1.

References: Alcalá \& Morales (1989).

\section{Panthera leo spelaea Goldfuss, 1810}

Sites: Solana de Zamborino.

References: Martín-Penela (1988).

Felidae indet.

Sites: Fuente Nueva-3.

References: Martínez-Navarro et al. (2010).

Hyaenidae Gray, 1821

Crocuta Erxleben, 1777

\section{Crocuta crocuta (Erxleben, 1777)}

Sites: Cúllar de Baza-1.

References: Ruíz-Bustos (1976), Alcalá \& Morales (1989).

Hyaena Brünnich, 1771

Hyaena brunnea Thunberg, 1820

Sites: Fonelas P-1.

References: Garrido (2006), Arribas et al. (2008).

Pachycrocuta Kretzoi, 1938

Pachycrocuta brevirostris (Aymard, 1846)

Sites: Fonelas P-1, Barranco León-5, Fuente Nueva-3, Venta Micena-1, Venta Micena-2.

References: Agustí et al. (1983, 1986), Pons-Moyà (1987), Arribas \& Palmqvist (1999), Martínez-Navarro et al. (2003, 2004, 2010), Garrido (2006), Arribas et al. (2008).

Hyaenidae indet.

Sites: Huéscar-1.

References: Alcalá \& Morales (1989).

Mustelidae Fischer de Waldheim, 1817

Meles Brisson, 1762

Meles iberica (Arribas \& Garrido, 2007)

Sites: Fonelas P-1.

References. Garrido (2006), Arribas \& Garrido (2007), Arribas et al. (2008).
Meles sp.

Sites: Venta Micena-2, Barranco León-5, Fuente Nueva-3. References: Agustí et al. (1983, 1986), Pons-Moyà (1987), Martínez-Navarro et al. (2003, 2004, 2010).

Pannonictis Kormos 1931

Pannonictis cf. nestii (Martelli, 1906)

Sites: Fuente Nueva-3.

References: Martínez-Navarro et al. (2010).

Mustelidae indet.

Sites: Fuente Nueva-3.

References: Martínez-Navarro et al. (2010).

Ursidae Gray, 1825

Ursus Linnaeus, 1758

Ursus etruscus Cuvier, 1823

Sites: Venta Micena-2.

References: Agustí et al. (1983, 1985, 1986), Pons-Moyà (1987), Martínez-Navarro (1991).

\section{Ursus sp.}

Sites: Barranco León-5, Fuente Nueva-3.

References: Martínez-Navarro et al. (2003, 2004, 2010).

ARTIODACTYLA Owen, 1848

Bovidae Gray, 1821

Alephis Gromolard, 1980

Alephis sp.

Sites: Baza-1.

References: Ros-Montoya et al. (in press).

Ammotragus Blyth, 1840

Ammotragus europaeus Moullé, Echassoux \& Martínez-Navarro, 2004.

Sites: Fuente Nueva-3.

References: Martínez-Navarro et al. (2010).

Bison Hamilton Smith, 1827

\section{Bison sp.}

Sites: Venta Micena-1, Venta Micena-2, Barranco León2, Barranco León-5, Fuente Nueva-3, Cúllar de Baza-1. References: Ruíz-Bustos (1976), Agustí et al. (1983, 1985, 1986, 1987b), Toro et al. (1984), Moya-Solá (1987), Azanza \& Morales (1989), Martínez-Navarro (1991), Martínez-Navarro et al. (2009, 2010). 


\section{Bison priscus Bojanus, 1827}

Sites: Solana de Zamborino.

References: Martín-Penela (1988).

Bos Linnaeus, 1758

Bos primigenius Bojanus, 1827

Sites: Solana de Zamborino.

References: Martín-Penela (1988).

Capra Linneo, 1758

Capra baetica Arribas \& Garrido, 2008

Sites: Fonelas P-1.

References: Garrido (2006), Arribas \& Garrido (2008).

Capra alba Moya-Sola, 1987

Sites: Venta Micena-1, Venta Micena-2, Barranco León-2. References: Agustí et al. (1983, 1986, 1987b), MartínezNavarro (1991).

Capreolus Gray, 1821

Capreolus capreolus Linnaeus, 1758

Sites: Solana de Zamborino.

References: Martín-Penela (1988).

Gazella Blainville, 1816

\section{Gazella borbonica Depéret, 1884}

Sites: Gorafe-4, Huélago-1, Fuente Nueva-1.

References: Agustí (1986), Moya-Solá et al. (1987), Azanza \& Morales (1989).

Gazellospira Pilgrim \& Schaub, 1939

Gazellospira torticornis (Aymard, 1854)

Sites: Huélago-1, Orce-2, Fonelas P-1.

References: Agustí et al. (1987b), Azanza \& Morales (1989), Garrido (2006).

Hemitragus Hodgson, 1841

Hemitragus albus (Crégut-Bonnoure \& Spassov, 2002)

Sites: Venta Micena-2, Barranco León-2, Fuente Nueva-3. References: Agustí et al. (1983, 1986), Martínez-Navarro (1991), Martínez-Navarro et al. (2003, 2004, 2010).

Hemitragus cf. albus (Crégut-Bonnoure \& Spassov, 2002)
Sites: Barranco León-5.

References: Martínez-Navarro et al. (2003, 2004, 2010).

Hemitragus sp.

Sites: Fonelas P-1.

References: Garrido (2006), Arribas et al. (2008).

Hesperidoceras Villalta \& Crusafont Pairó, 1956

Hesperidoceras merlae Villalta \& Crusafont Pairó, 1956

Sites: Huélago-1.

References: Azanza \& Morales (1989).

Hesperidoceras sp.

Sites: Barranco del Paso, Venta Micena-2, Barranco León5, Fuente Nueva-3.

References: Agustí et al. (1983, 1986), Toro et al. (1984), Martínez-Navarro (1991), Gibert et al. (1992), MartínezNavarro et al. $(2003,2010)$.

Leptobos Rütimeyer, 1877

Leptobos cf. elatus (Pomel, 1853)

Sites: Huélago-1.

References: Azanza \& Morales (1989).

$$
\text { cf. Leptobos sp. }
$$

Sites: Orce-6.

References: Agustí et al. (1987b, 1997).

Praeovibos mediterraneus Crégut-Bonnoure, 2002

Sites: Venta Micena.

References: Crégut-Bonnoure (2002).

Praeovibos sp.

Sites: Fonelas P-1, Barranco de los Conejos.

References: Garrido (2008b), Agustí et al. (2013).

Soergelia Schaub, 1951

Soergelia minor Moyà-Solà, 1987

Sites: Venta Micena-1, Venta Micena-2, Barranco León-2. References: Agustí et al. (1983, 1986, 1987b), Moya-Solá (1987), Martínez-Navarro, (1991).

\section{Soergelia sp.}

Sites: Barranco León-5, Fuente Nueva-3, Fonelas P-1. References: Martínez-Navarro (2003, 2010), Garrido (2006), Arribas et al. (2008). 
Cervidae Goldfuss, 1820

Alces Gray, 1821

Alces sp.

Sites: Cortes de Baza.

References: Aguirre (1958).

Cervus Linnaeus, 1758

Cervus acoronatus Beninde, 1937

Sites: Cúllar de Baza-1.

References: Ruíz-Bustos (1976), Toro et al. (1984).

Cervus elaphus Linnaeus, 1758

Sites: Solana de Zamborino.

References: Martín-Penela (1988).

Croizetoceros Heintz, 1970

Croizetoceros ramosus (Croizet \& Jobert, 1828)

Sites: Huélago-1, Fonelas P-1.

References: Azanza \& Morales (1989), Garrido (2006).

Dama Linnaeus, 1758

Dama sp.

Sites: Solana de Zamborino.

References: Martín-Penela (1988).

Dolichodoryceros Kahlke, 1956

Dolichodoryceros savini (Dawkins, 1887)

Sites: Cúllar de Baza-1.

References: Azanza \& Morales (1989).

Eucladoceros Falconer, 1868

Eucladoceros cf. senezensis (Depéret, 1910)

Sites: Huélago-1.

References: Azanza \& Morales (1989).

Eucladoceros sp.

Sites: Fonelas P-1.

References: Garrido (2006).

Metacervoceros Dietrich, 1938

Metacervocerus rhenanus (Dubois, 1904)

Sites: Fonelas P-1, Barranco del Paso, Venta Micena-2, Barranco León-5, Fuente Nueva-3.

References: Agustí et al. (1983, 1985, 1986), Menéndez
(1987), Martínez-Navarro (1991), Gibert et al. (1992), Martínez-Navarro et al. (2003, 2004), Garrido (2006), Abbazzi (2010).

Praemegaceros Portis, 1920

Praemegaceros cf. solilhacus (Robert, 1829)

Sites: Huéscar-1.

References: Azanza \& Morales (1989).

Praemegaceros verticornis Ruiz-Bustos, 1976

Sites: Venta Micena-2, Barranco León-5, Fuente Nueva-3, Cúllar de Baza-1.

References: Ruíz-Bustos (1976), Agustí et al. (1983, 1985, 1986), Martínez-Navarro (1991), Martínez-Navarro et al. (2003, 2004), Abbazzi (2010).

Cervidae indet.

Sites: Baza-1.

References: Ros-Montoya et al. (in press).

Giraffidae Gray, 1821

Mitilanotherium Samson \& Radulesco, 1966

Mitilanotherium inexpectatum Samson \& Radulesco, 1966

Sites: Huélago.

References: Van der Made \& Morales (2011).

\section{Mitilanotherium sp.}

Sites: Fonelas P-1.

References: Garrido \& Arribas (2008b).

Hippopotamidae Gray, 1821

Hippopotamus Linnaeus, 1758

Hippopotamus antiquus Desmarest, 1822

Sites: Venta Micena-2, Barranco León-2, Barranco León5, Fuente Nueva-3, Solana de Zamborino.

References: Alberdi \& Ruíz-Bustos (1985), Martín-Penela (1988), Martínez-Navarro (1991), Martínez-Navarro et al. (2003, 2004, 2010).

\section{Hippopotamus major Cuvier 1824}

Sites: Huéscar-1

References: Mazo (1989a).

Suidae Gray, 1821

Potamochoerus Gray, 1854

Potamochoerus magnus $\mathrm{n} . \mathrm{sp}$.

Sites: Fonelas P-1.

References: Garrido (2006), Arribas et al. (2008). 
Sus Linnaeus, 1758

Sus arvernensis Linnaeus, 1758

Sites: Gorafe 4.

References: Van der Made (1989a, 1989b).

Sus scrofa Linnaeus, 1758

Sites: Solana de Zamborino.

References: Martín-Penela (1988).

Sus cf. scrofa Linnaeus, 1758

Sites: Cúllar de Baza-1.

References: Ruíz-Bustos (1976), Azanza \& Morales (1989).

PERISSODACTYLA Owen, 1848

Equidae Gray 1821

Equus Linneo, 1758

Equus altidens altidens von Reichenau, 1915

Sites: Huéscar-1, Cúllar de Baza-1.

References: Alberdi \& Ruíz-Bustos (1989), Alberdi et al. (1998).

Equus altidens granatensis Alberdi \& Ruiz Bustos, 1985

Sites: Venta Micena-2, Láchar, Fuensanta, Fuente Nueva-3 y Barranco León-5.

References: Marín (1987), Alberdi \& Ruíz-Bustos (1989), Alberdi et al. (1998), Alberdi (2010).

Equus caballus ssp. Linnaeus, 1758

Sites: Cúllar de Baza-2.

References: Alberdi \& Ruíz-Bustos (1989).

Equus caballus torralbae Prat, 1977

Sites: Solana de Zamborino.

References: Martín-Penela (1988), Alberdi \& Ruíz-Bustos (1989).

Equus cf. major Depéret, 1893

Sites: Fonelas P-1.

References: Garrido (2008c).

Equus stenonis Cocchi, 1867

Sites: Orce-2, Orce-6, Fuente Nueva-2, Venta Micena-2. References: Agustí et al. (1983, 1985, 1986), MartínezNavarro (1991).

Equus stenonis stenonis Cocchi, 1867
Sites: Cortes de Baza-1, Cortijo de Tapia-1.

References: Alberdi \& Ruíz-Bustos (1989), Alberdi et al. (1998).

Equus stenonis aff. vireti Prat, 1964

Sites: Fuente Nueva-1.

References: Moyà-Solà et al. (1987).

Equus stenonis ssp. Cocchi, 1867

Sites: Barranco de los Conejos, Barranco León-2.

References: Agustí et al. (1987b, 2013).

Equus livenzovensis Bajgusheva, 1978

Sites: Huélago-1.

References: Alberdi et al. (1998).

Equus cf. livenzovensis Bajgusheva, 1978

Sites: Barranco de las Cañuelas-1.

References: Alberdi \& Ruíz-Bustos (1989), Alberdi et al. (1998).

Equus suessenbornensis Wüst, 1900

Sites: Barranco León-5, Fuente Nueva-3, Huéscar-1, Cúllar de Baza-1.

References: Alberdi \& Ruíz-Bustos (1989), Alberdi (2010).

\section{Equus sp.}

Sites: Barranco del Paso.

References: Gibert et al. (1992).

Hipparion De Christol, 1832

Hipparion sp.

Sites: Barranco de Quebradas-1, Baza-1.

References: Alberdi \& Ruíz Bustos (1989), Ros-Montoya et al. (in press).

Rhinocerotidae Gray, 1821

Stephanorhinus Kretzoi, 1942

Stephanorhinus etruscus (Falconer, 1859)

Sites: Fonelas P-1, Barranco del Paso, Huéscar-1, Cúllar de Baza-1, Venta Micena.

References: Santafé-Llopis \& Casanovas-Cladellas (1987), Cerdeño (1989), Gibert et al. (1992), Garrido (2008c).

Stephanorhinus cf. etruscus (Falconer, 1859)

Sites: Huélago-1.

References: Cerdeño (1989).

Stephanorhinus hemitoechus (Falconer, 1868) 
Sites: Solana de Zamborino.

References: Martín-Penela (1988).

Stephanorhinus hundseimensis (Toula, 1902)

Sites: Venta Micena-1, Venta Micena-2, Barranco León5, Fuente Nueva-3.

References: Agustí et al. (1983, 1986), Martínez-Navarro (1991), Lacombat (2010).

\section{Stephanorhinus kirchbergensis (Jäger, 1839)}

Sites: Solana de Zamborino.

References: Martín-Penela (1988).

\section{cf. Stephanorhinus jeanvireti Guérin, 1972}

Sites: Baza-1.

References: Ros-Montoya et al. (in press).

PROBOSCIDEA Illiger, 1811

Elephantidae Gray, 1821

Elephas Linnaeus, 1758

Elephas antiquus Falconer \& Cautley, 1847

Sites: Huéscar-1, Solana de Zamborino.

References: Mazo et al. (1985), Martín-Penela (1988), Mazo (1989b), Ros-Montoya (2010), Ros-Montoya et al. (2010).

Mammuthus Brookes, 1828

Mammuthus meridionalis (Nesti, 1825)

Sites: Huélago-1, Fonelas P-1, Zújar-14, Barranco del Paso, Cortijo de las Sabinas, Cortes de Baza, Venta Micena-2, Barranco León-2, Barranco León-5, Fuente Nueva-3, Orce-3, Láchar.

References: Aguirre (1958), Agustí et al. (1983, 1986, 2001), Mazo et al. (1985), Gibert et al. (1992), Agustí \& Oms (1998), Mazo (1989a), Martínez-Navarro (1991), Martínez-Navarro et al. (2003, 2004), Garrido (2006), RosMontoya (2010), Ros-Montoya et al. (2010).

Mammuthus trogontherii (Pohlig, 1885)

Sites: Cúllar de Baza-1, Solana de Zamborino. References: Martín-Penela (1988), Mazo (1989b), RosMontoya (2010), Ros-Montoya et al. (2010).

Mammutidae Hay, 1922

Mammut Blumenbach, 1799

Mammut borsoni Blumenbach, 1799

Sites: Baza-1.

References: Ros-Montoya (2010), Ros-Montoya et al. (2010, in press), Martínez-Navarro et al. (2015).
Tetralophodontidae Vaufrey, 1958

Anancus Aymard, 1855

Anancus arvernensis (Croizet \& Jobert, 1828)

Sites: Canal de San Clemente, Barranco de Quebradas-1, Baza-1, Zújar-11.

References: Aguirre (1958), Mazo (1989b), Agustí \& Oms (1998), Agustí et al. (2001), Ros-Montoya (2010), RosMontoya et al. (2010, in press), Martínez-Navarro et al. (2015).

\section{CONCLUSIONS}

More than 300 vertebrate species have been identified in the Guadix-Baza Basin. Among rodents, the families Muridae and Arvicolidae are the most abundant throughout the whole succession. The diversity of murids decreases in the late Pliocene, from MN 15 to MN 16. In contrast, arvicolids first appear at MN 15 in the basin and their diversity increases during the Early Pleistocene. We can also quote the significant presence of members of the families Cricetidae, Gerbillidae, Sciuridae and Trilophomydae in the units MN 13, MN 14 and MN 15, becoming absent in the latest Pliocene and the Early Pleistocene. However, the cricetids reappear at the beginning of the Middle Pleistocene (MmQ 4). The presence of members of the family Castoridae at MN 16 and Hystricidae at MmQ 2 and MmQ 3a is also interesting.

Among insectivores, the three familes Talpidae, Soricidae and Erinaceidae are present. The most abundant family is Soricidae, present all along the sequence. This is in contrast with talpids, which are absent in the associations from the late Miocene (MN 13) and the Middle Pleistocene (MmQ 4). Talpids are present in the Pliocene (MN 14, MN 15, MN 16) and the Early Pleistocene (MmQ 2). Erinaceids are present in the late Miocene (MN 10, MN 13), early Pliocene (MN 14, MN 15) and late Early Pleistocene (MN 17, MmQ 3a).

The best-represented epoch in the Guadix-Baza Basin is the Pliocene (MN 14 to MN 16), with 41 sites, particularly the MN 15 (17 sites). This is followed by the Early Pleistocene (MN 17 and MmQ1 to MmQ 3), with 30 sites. The MN 17 (9 sites) and the MmQ 1 (9 sites) are the best represented biochronological units of this time interval. The late Miocene is represented by 2 localities assigned to the MN 10 unit, and 7 sites assigned to the MN 13 unit, while the Middle Pleistocene (MmQ 4) is represented by 5 sites. Due to its paleontological richness and stratigraphic continuity, the succession in the GuadixBaza Basin represents a unique case in Western Europe and a reference for correlation with other areas. 


\section{ACKNOWLEDGEMENTS}

We are glad to devote this paper to Prof. Pascual Rivas, who did so much to enhance the paleontological research in the Guadix-Baza Basin. We are also grateful to a number of persons who revised the content of this paper: HuguesAlexandre Blain, Angel Blanco, Marc Furió, Bienvenido Martínez-Navarro. This paper is part of the projects CGL2016-80000-P (MINECO, Spain) and SGR2014901 (CERCA Program, Generalitat de Catalunya). Pedro Piñero is supported by a FPU Predoctoral Fellowship (FPU12/02668). The authors thank to the editor Francisco J. Rodríguez-Tovar, and the reviewers Raef MinwerBarakat and Antonio García-Alix for their valuable comments and suggestions on the manuscript.

\section{REFERENCES}

Abbazzi, L. 2010. La fauna de Cérvidos de Barranco León y Fuente Nueva 3. In: Ocupaciones Humanas en el Pleistoceno Inferior y Medio de la Cuenca de GuadixBaza, Memoria Cientifica (eds Toro, I., Martínez-Navarro, B. \& Agustí, J.). Junta de Andalucía, Consejería de Cultura. E.P.G., Arqueología Monográfías, 273-290.

Aguirre, E. 1958. Novedades paleomastológicas de la Depresión de Granada y estratigrafía de su borde NE. Estudios Geológicos, 14, 107-120.

Agustí, J. 1985. Bioestratigrafía de los depósitos PlioPleistocenos de la depresión de Guadix-Baza. (Prov. Granada). Paleontologia i Evolució, 18, 13-18.

Agustí, J. 1986. Synthèse biostratigraphique du PlioPléistocène de Guadix-Baza (Province de Granada, sudest de l'Espagne). Geobios, 19, 505-510; doi: 10.1016/ S0016-6995(86)80007-9.

Agustí, J. 1990. The plio-pleistocene arvicolid succession in southern and eastern Spain. In: International Symposium on the Evolution, Phylogeny and Biostratigraphy of Arvicolids (Rodentia, Mammalia) (eds Fejfar, O. \& Heinrich, W.D.). Geological Survey, Praha, 21-32.

Agustí, J. 1991. The Allophaiomys Complex in Southern Europe. Geobios, 25, 133-144; doi: 10.1016/S00166995(09)90043-2.

Agustí, J. \& Madurell, J. 2003. Los arvicólidos (Muroidea, Rodentia, Mammalia) del Pleistoceno inferior de Barranco León y Fuente Nueva 3 (Orce, Granada). Datos preliminares. In: El Pleistoceno Inferior de Barranco León y Fuente Nueva 3, Orce (Granada). Memoria Cientifica Campañas 1999-2002 (eds Toro, I., Agustí, J. \& MartínezNavarro, B.). Junta de Andalucía, Consejería de Cultura. E.P.G., Arqueología Monográfico, 137-146.

Agustí, J. \& Martín-Suárez, E. 1994. Síntesis biostratigráfica de la Cuenca de Guadix-Baza. Comunicaciones de las X Jornadas de Paleontología. Sociedad Española de Paleontología. Madrid, 8-9.
Agustí, J. \& Oms, O. 1998. The mammal succession in the Negratín Clay (Jabalcón area). Resúmenes de comunicaciones: Excursion to the Guadix-Baza Basin, Euromam. Junta de Andalucía, 26.

Agustí, J., Anadón, P., Gibert, J., Juliá, R., Martín-Suárez, E., Menéndez, E., Moyà-Solà, S., Pons-Moyá, J., Rivas, P. \& Toro, I. 1983. Estratigrafía y paleontología del Pleistoceno inferior de Venta Micena (Orce, depresión de GuadixBaza, Granada): Resultados preliminares. Paleontologia i Evolució, 18, 19-38.

Agustí, J., Arbiol, S. \& Martín-Suárez, E. 1987c. Roedores y lagomorfos (Mammalia) del Pleistoceno inferior de Venta Micena (Guadix-Baza, Granada). Paleontologia $i$ Evolució. Mem. Esp., 1, 95-107.

Agustí, J., Blain, H.A., Furió, M., De Marfà, R. \& SantosCubedo, A. 2010. The early Pleistocene small vertebrate succession from the Orce region (Guadix-Baza Basin, SE Spain) and its bearing on the first human occupation of Europe. Quaternary International, 223-224, 162-169; doi: 10.1016/j.quaint.2009.12.011.

Agustí, J., Blain, H-A., Furió, M., De Marfà, R., MartínezNavarro, B. \& Oms, O. 2013. Early Pleistocene environments and vertébrate dispersals in Western Europe: The case of Barranco de los Conejos (Guadix-Baza Basin, SE Spain). Quaternary International, 295, 59-68; doi: 10.1016/j.quaint.2012.02.004.

Agustí, J., Blain, H-A., Lozano-Fernández, I., Piñero, P., Oms, O., Furió, M., Blanco, A., López-García, J.M. \& Sala, R. 2015b. Chronological and environmental context of the first hominin dispersal into Western Europe: The case of Barranco León (Guadix-Baza Basin, SE Spain). Journal of Human Evolution, 87, 87-94; doi: 10.1016/j. jhevol.2015.02.014.

Agustí, J., De Marfà, R. \& Santos-Cubedos, A. 2010. Roedores y Lagomorfos (Mammalia) del Plesitoceno inferior de Barranco León y Fuente Nueva 3 (Orce, Granada). In: Ocupaciones Humanas en el Pleistoceno Inferior y Medio de la Cuenca de Guadix-Baza, Memoria Cientifica (eds Toro, I., Martínez-Navarro, B. \& Agustí, J.). Junta de Andalucía, Consejería de Cultura. E.P.G., Arqueología Monográfico, 95-108.

Agustí, J., Gibert, J., Moyà, S. \& Vera, J.A. 1985. NeogeneQuaternary boundary in the Continental Sediments of the Guadix-Baza basin (Southeastern Spain). VII Congress Regional Committee on Mediterranean Neogene Stratigraphy. Budapest, Abstracts, 50-52.

Agustí, J., Lozano-Fernández, I., Oms, O., Piñero, P., Furió, M., Blain, H-A., López-García, J.M. \& MartínezNavarro, B. 2015a. Early to Middle Pleistocene rodent biostratigraphy of the Guadix-Baza Basin (SE Spain). Quaternary International, 389, 139-147; doi: 10.1016/j. quaint.2014.11.005.

Agustí, J., Moyà-Solà, S. \& Pons-Moyà, J. 1986. Venta Micena (Guadix-Baza Basin, southeastern Spain): its place in the Plio-Pleistocene mammal succession in Europe. Geologica Romana, 25, 33-62.

Agustí, J., Moyà-Solà, S., Martín-Suárez, E. \& Marín, M. 1987b. Fauna de mamíferos en el Pleistoceno inferior 
de la región de Orce (Granada, España). Paleontologia $i$ Evolució, Mem. Esp., 1, 73-86.

Agustí, J., Moyà Solà, S. \& Pons Moyà, J. 1987a. La sucesión de Mamíferos en el Pleistoceno inferior de Europa: proposición de una nueva escala bioestratigráfica. Paleontologia i Evolució, Mem. Esp., 1, 287-295.

Agustí, J., Oms, O., Garces, M. \& Parés, J.M. 1997. Calibration of the late Pliocene-Early Pleistocene transition in the continental beds of the Guadix-Baza basin (Southeastern Spain). Quaternary International, 40, 93-100; doi: 10.1016/S1040-6182(96)00065-1.

Agustí, J., Oms, O. \& Remacha, E. 2001. Long PlioPleistocene terrestrial record of climate change and mammal turnover in southern Spain. Quaternary Research, 56, 411-418; doi: 10.1006/qres.2001.2269.

Alberdi, M.T. 2010. Estudio de los caballos de los yacimientos de Fuente Nueva-3 y Barranco León-5 (Granada). In: Ocupaciones Humanas en el Pleistoceno Inferior y Medio de la Cuenca de Guadix-Baza, Memoria Cientifica (eds Toro, I., Martínez-Navarro, B. \& Agustí, J.). Junta de Andalucía, Consejería de Cultura E.P.G. Arqueología Monográfico, 291-306.

Alberdi, M.T. \& Bonadonna, F.P. 1989. Geología y Paleontología de la Cuenca de Guadix-Baza. Trabajos sobre el Neógeno-Cuaternario. Museo Nacional de Ciencias Naturales, CSIC, Madrid, 355 pp.

Alberdi, M.T. \& Ruíz-Bustos, A. 1985. Descripción y significado bioestratigráfico del Equus e Hipopótamo en el yacimiento de Venta Micena (Granada). Estudios Geológicos, 41, 251-261.

Alberdi, M.T. \& Ruíz-Bustos, A. 1989. Taxonomía y bioestratigrafía de Equidae (Mammalia, Perissodactyla) en la Cuenca de Guadix-Baza (Granada). In: Geología y Paleontología de la Cuenca de Guadix-Baza Trabajos sobre el Neógeno-Cuaternario (eds Alberdi, M.T. \& Bonadonna, F.). Museo Nacional de Ciencias Naturales, CSIC, Madrid, 11, 239-271.

Alberdi, M.T., Alonso, M.A., Cerdeño, E. \& Ruíz-Bustos, A. 1989. Investigaciones paleontológicas realizadas en la Cuenca Guadix-Baza entre 1983 y 1987. In: Geología y Paleontología de la Cuenca de Guadix-Baza Trabajos sobre el Neógeno-Cuaternario (eds Alberdi, M.T. \& Bonadonna, F.). Museo Nacional de Ciencias Naturales, CSIC, Madrid, 11, 13-52.

Alberdi, M.T., Ortiz-Jaureguizar, E. \& Prado, J.L. 1998. A quantitative review of European stenonoid horses. Journal of Paleontology, 72, 371-387.

Alcalá, L. \& Morales, J. 1989. Los carnívoros del Pleistoceno medio de Cúllar Baza-1 y Huéscar-1 (Cuenca de GuadixBaza). In: Geología y Paleontología de la Cuenca de Guadix-Baza Trabajos sobre el Neógeno-Cuaternario (eds Alberdi, M.T. \& Bonadonna, F.). Museo Nacional de Ciencias Naturales, CSIC, Madrid, 11, 215-223.

Anchelergues Tarraco, A., Martín-Suárez, E. \& Freudenthal, M. 2015: Muridae (Rodentia) from the early Pleistocene of Loma Quemada-1 (Granada, Spain). Palaeobiodiversity and Palaeoenvironments, 95, 347-352; doi: 10.1007/ s12549-015-0189-9.
Arribas, A. \& Palmqvist, P. 1999. El modelo de actividad de Pachycrocuta en Venta Micena: implicación de los hiénidos en el registro paleomastológico Plio-Pleistoceno español. Temas Geológico-Mineros ITGE, 26, 173-181.

Arribas, A. \& Garrido, G. 2007. Meles iberica n. sp., a new Eurasian badger (Mammalia, carnivora, mustelidae) from Fonelas P-1 (Plio-Pleistocene boundary, Guadix basin, Granada, Spain). Comptes Rendus Palevol, 6, 545-555; doi: 10.1016/j.crpv.2007.06.002.

Arribas, A. \& Garrido, G. 2008. Los representantes más antiguos del género Capra (Bovidae, Artiodactyla, Mammalia) en el registro euroasiático (Fonelas P-1, Cuenca de Guadix, España). In: Vertebrados del Plioceno Superior Terminal en el Suroeste de Europa: Fonelas P-1 $y$ el Proyecto Fonelas (ed. Arribas, A.). Cuadernos del Museo Geominero 10, pp. 461-473.

Arribas, A., Baeza, E., Bermúdez, D., Blanco, S., Durán, J.J., Garrido, G., Gumiel, J.C., Hernández, R., Soria, J.M. \& Viseras, C. 2004. Nuevos registros paleontológicos de grandes mamíferos en la Cuenca de Guadix-Baza (Granada): aportaciones del Proyecto Fonelas al conocimiento sobre las faunas continentales del PliocenoPleistoceno europeo. Boletín Geológico y Minero, 115, 567-581.

Arribas, A., Garrido, G., Solano, J.G., Hernández, R., Carroza, J.A., Lorenzo, C., Gumiel, J.C., Baeza, E., Lozano, R.P., De Frutos, C., Durán, J.J., Laplana, C., López-Martínez, J., Soria, J.M., Garcés, M., Díez, J.C., Navazo, M., Terradillos, M., Alonso, R., Viseras, C., Pla, S., Carrión, J.S., Fernández, S. \& Blain, H.A. 2008. Vertebrados del Plioceno Superior Terminal en el Suroeste de Europa: Fonelas P-1 y el Proyecto Fonelas. Cuadernos del Museo Geominero, 10, $607 \mathrm{pp}$.

Azanza, B. \& Morales, J. 1989. Los artiodáctilos de los yacimientos de Huélago, Huéscar 1 y Cúllar-Baza 1 (cuenca de Guadix-Baza, Granada). In: Geología y Paleontología de la Cuenca de Guadix-Baza Trabajos sobre el Neógeno-Cuaternario (eds Alberdi, M.T. \& Bonadonna, F.). Museo Nacional de Ciencias Naturales, CSIC, Madrid, 11, 289-316.

Bailon, S. 2010. Quelonios fósiles del yacimiento de Barranco León (Pleistoceno Inferior, Orce, Granada, España). In: Ocupaciones Humanas en el Pleistoceno Inferior y Medio de la Cuenca de Guadix-Baza, Memoria Científica (eds Toro, I., Martínez-Navarro, B. \& Agustí, J.). Junta de Andalucía. Consejería de Cultura, E.P.G. Arqueología Monográfico, 185-195.

Barbadillo, L.J. 1989. Los reptilia (Sauria y Amphisbaenia) de los yacimientos Plio-Pleistocénicos de la Cuenca de Guadix-Baza. In: Geología y Paleontología de la Cuenca de Guadix-Baza Trabajos sobre el Neógeno-Cuaternario (eds Alberdi, M.T. \& Bonadonna, F.). Museo Nacional de Ciencias Naturales, CSIC, Madrid, 11, 151-166.

Blain, H.A. 2003. Los anfibios y reptiles de los yacimientos del Pleistoceno inferior de Barranco León y Fuente Nueva 3, (Orce, Granada, España). In: El Pleistoceno Inferior de Barranco León y Fuente Nueva 3, Orce (Granada). Memoria Cientifica Campañas 1999-2002 (eds Toro, I., 
Agustí, J. \& Martínez-Navarro, B.). Junta de Andalucía. Consejería de Cultura, E.P.G. Arqueología Monográfico, 159-172.

Blain, H.A. 2009. Contribution de la paléoherpétofaune (Amphibia y Squamata) à la connaissance de l'évolution du climat et du paysage du Pliocène supérieur au Pléistocène moyen d'Espagne. Treballs del Museu De Geologia Barcelona, 16, 39-170.

Blain, H.A. \& Bailon, S. 2010. Anfibios y Escamosos del Pleistoceno inferior de Barranco León y Fuente Nueva 3 (Orce, Andalucía, España). In: Ocupaciones Humanas en el Pleistoceno Inferior y Medio de la Cuenca de Guadix-Baza, Memoria Cientifica (eds Toro, I., MartínezNavarro, B. \& Agustí, J.). Junta de Andalucía. Consejería de Cultura, E.P.G. Arqueología Monográfico, 165-183.

Bruijn, H. de. 1974. The Ruscinian rodent succession in Southern Spain and its implications for the biostratigraphic correlation of Europe and North Africa. Senckenbergiana lethaea, 55, 435-443.

Castillo C. \& J. Agustí. 1996. Early Pliocene rodents (Mammalia) from Asta Regia (Jerez Basin, Southwestern Spain). Koninklijke Nederlandse Akademie van Wetenschappen, 99, 25-43.

Cerdeño, E. 1989. Rhinocerotidae (Mammalia, Perissodactyla) en la Cuenca de Guadix-Baza. In: Geología y Paleontología de la Cuenca de Guadix-Baza Trabajos sobre el NeógenoCuaternario (eds Alberdi, M.T. \& Bonadonna, F.). Museo Nacional de Ciencias Naturales, CSIC, Madrid, 11, 273-287.

Crégut-Bonnoure, E. 2002. Les Ovibovini, Caprini et Ovini (Mammalia, Artiodactyla, Bovidae, Caprinae) du PlioPléistocène d'Europe: systématique, évolution et biochronologie. 429 pp. Ph.D. Thesis (unpublished), Université Claude Bernard, Lyon.

De Marfà, R. 2007, Microfauna del Pleistoceno Inferior de Barranco León y Fuente Nueva 3 (Orce, Granada, España): Estudio preliminar). Actas del III Encuentro de Jóvenes Investigadores en Paleontología, 45-56.

De Marfà, R. 2009. Els Lagomorfs (O. Lagomorpha, cl. Mammalia) del Pliocè i el Pleistocè Europeus. Jacimients estudiats. Ph.D. Thesis (unpublished), Universitat de Barcelona.

Doadrio, I. \& Casado, P. 1989. Nota sobre la sobre la ictiofauna continental de los yacimientos de la Cuenca Guadix-Baza (Granada). In: Geología y Paleontología de la Cuenca de Guadix-Baza Trabajos sobre el NeógenoCuaternario (eds Alberdi, M.T. \& Bonadonna, F.). Museo Nacional de Ciencias Naturales, CSIC, Madrid, $11,139-150$.

Fèlix, J. \& Montori, A. 1987. Los anfibios y los reptiles del Plio-Pleistoceno de la depresión de Guadix-Baza (Granada). Paleontologia i Evolució, Mem. Esp., 1, 283-286.

Fernández, J., Soria, J.M. \& Viseras, C. 1996a. Stratigraphic architecture of the Neogene basins in the central sector of the Betic Cordillera (Spain): tectonic control and base-level changes. In: Tertiary Basins of Spain: The Stratigraphic Record of Crustal Kinematics (eds Friend, P.F. \& Dabrio, C.J.). Cambridge University Press, Cambridge, 353-365.
Fernández, J., Viseras, C. \& Soria, J.M. 1996b. PliocenePleistocene continental infilling of the Granada and Guadix basins (Betic Cordillera, Spain): the influence of allocyclic and autocyclic processes on the resultant stratigraphic organization. In: Tertiary Basins of Spain: The Stratigraphic Record of Crustal Kinematics (eds Friend, P.F. \& Dabrio, C.J.). Cambridge University Press, Cambridge, 366-371.

Freudenthal, M., Mein, P. \& Martín-Suárez, E. 1998. Revision of Late Miocene and Pliocene Cricetinae (Rodentia, Mammalia) from Spain and France. Treballs del Museu de Geologia de Barcelona, 7, 11-93.

Furió, M. 2003. Los insectívoros (mammalia) del Pleistoceno inferior de Fuente Nueva 3 y Barranco León (Orce, Granada). In: El Pleistoceno Inferior de Barranco León y Fuente Nueva 3, Orce (Granada). Memoria Cientifica Campañas 1999-2002 (eds Toro, I., Agustí, J. \& MartínezNavarro, B.). Junta de Andalucía, Consejería de Cultura, E.P.G. Arqueología Monográfico, 147-158.

Furió, M. 2007. Los insectívoros (Soricomorpha, Erinaceomorpha, Mammalia) del Neógeno Superior del Levante Ibérico. Cuenca de Guadix-Baza. 341 pp. Ph.D. Thesis (unpublished), Universitat Autónoma de Barcelona, Barcelona.

Furió, M. 2010. Contribución al conocimiento de los Insectívoros (Insectívora, Mammalia) del Pleistoceno inferior de Barranco León y Fuente Nueva 3 (Orce, Granada). In: Ocupaciones Humanas en el Pleistoceno Inferior y Medio de la Cuenca de Guadix-Baza, Memoria Cientifica (eds Toro, I., Martínez-Navarro, B. \& Agustí, J.). Junta de Andalucía, Consejería de Cultura, E.P.G. Arqueología Monográfico, 141-164.

Furió, M., Santos-Cubedo, A., Minwer-Barakat, R. \& Agustí, J. 2007. Evolutionary history of the African Soricid Myosorex (Insectivora, Mammalia) out of Africa. Journal of Vertebrate Paleontology, 27, 1018-1024.

García-Alix, A., Minwer-Barakat, R., Martín-Suárez, E. \& Freudenthal, M. 2007. New data on Mio-Pliocene Sciuridae (Rodentia, Mammalia) from Southern Spain. Comptes Rendus Palevol, 6, 269-279; doi: 10.1016/j. crpv.2007.02.001.

García-Alix, A., Minwer-Barakat, R., Martín-Suárez, E. \& Freudenthal, M. 2008. Cricetidae and Gliridae (Rodentia, Mammalia) from the Miocene and Pliocene of Southern Spain. Scripta Geologica, 136, 1-37.

García-Alix, A., Minwer-Barakat, R., Martín-Suárez, E., Freudenthal, M., Aguirre, J. \& Kaya, F. 2016. Updating the Europe-Africa small mammal exchange during the late Messinian. Journal of Biogeography, 43, 1336-1348; doi: 10.1111/jbi.12732.

Garrido, G. 2006. Paleontología sistemática de grandes mamíferos del yacimiento del Villafranquiense superior de Fonelas P-1 (Cuenca de Guadix, Granada). Ph.D. Thesis (unpublished), Universidad Complutense de Madrid, Madrid.

Garrido, G. 2008a. El registro de Vulpes alopecoides (forsythmajor, 1877), Canis etrucus forsyth-major, 1877 y Canis cf. falconeri forsyth-major, 1877 (Canidae, Carnivora, 
Mammalia) en Fonelas P-1 (Cuenca de Guadix, Granada). In: Vertebrados del Plioceno Superior Terminal en el Suroeste de Europa: Fonelas P-1 y el Proyecto Fonelas (ed. Arribas, A.). Cuadernos del Museo Geominero, 10, 65-84.

Garrido, G. 2008b. Praeovibos sp. (Bovidae, Artiodactyla, Mammalia): primera cita del género en el Plioceno superior terminal de Eurasia (Fonelas P-1, Cuenca de Guadix-Baza). In: Vertebrados del Plioceno Superior Terminal en el Suroeste de Europa: Fonelas P-1 y el Proyecto Fonelas (ed. Arribas, A.). Cuadernos del Museo Geominero, 10, 475-488.

Garrido, G. 2008c. Los perisodáctilos [Equus cf. major Depéret, 1893 y Stephanorhinus etruscus (Falconer, 1859)] del yacimiento Plioceno superior terminal de Fonelas P-1 (Cuenca de Guadix, Granada). In: Vertebrados del Plioceno Superior Terminal en el Suroeste de Europa: Fonelas P-1 y el Proyecto Fonelas (ed. Arribas, A.). Cuadernos del Museo Geominero, 10, 553-595.

Garrido, G. \& Arribas, A. 2008b. Sobre la única población conocida del jiráfido Mitilanotherium (Giraffidae, Artiodactyla, Mammalia) en el Plioceno Superior terminal de Europa occidental (Fonelas P-1, Cuenca de Guadix, Granada). In: Vertebrados del Plioceno Superior Terminal en el Suroeste de Europa: Fonelas P-1 y el Proyecto Fonelas (ed. Arribas, A.). Cuadernos del Museo Geominero, 10, 397-411.

Gibert, J., Arribas, A., Terán, J. \& Palomar, J. 1992. Contexto geológico del Barranco del Paso (Orce, Granada). In: Presencia Humana en el Pleistoceno Inferior de Granada y Murcia Proyecto Orce-Cueva Victoria (1988-1992) (ed. Gibert, J.). Ayuntamiento de Orce, 203-218.

Gibert, L., Scott, G., Martin, R. \& Gibert, J. 2007. The early to middle Pleistocene boundary in the Baza Basin. Quaternary Science Reviews, 26, 2067-2089; doi: 10.1016/j.quascirev.2007.06.012.

Guerra Merchán, A. \& Ruiz Bustos, A. 1991. Geología y Paleontología del Plioceno continental en el sector de Baza (Cuenca de Guadix-Baza, Cordilleras Béticas). Geogaceta, 10, 24-28.

Guerra Merchán, A. \& Ruiz Bustos, A. 1992. Nuevos datos bioestratigráficos de los materiales continentales del sector suroriental de la Cuenca de Guadix-Baza. El yacimiento de Caniles. Geogaceta, 11, 76-78.

Guerra Merchán, A., Ruiz Bustos, A. \& Martín-Penela, A.J. 1991: Geología y fauna de los yacimientos de Colorado 1, Colorado 2, Aljibe 2 y Aljibe 3. (Cuenca de Guadix-Baza, Cordilleras Béticas). Geogaceta, 9, 99-102.

Hüsing, S.K., Oms, O., Agustí, J., Garcés, M., Kouwenhoven, T.J., Krijgsman, W. \& Zachariasse, W.-J. 2010. On the late Miocene closure of the Mediterranean-Atlantic gateway through the Guadix basin (southern Spain). Palaeogeography, Palaeoclimatology, Palaeoecology, 291, 167-179; doi: 10.1016/j.palaeo.2010.02.005.

Hüsing, S.K., Oms, O., Agustí, J., Garcés, M., Kouwenhoven, T.J., Krijgsman, W., Zachariasse, W.-J. 2012. On the Late Miocene continentalization of the Guadix Basin: More evidence for a major Messinian hiatus. Geobios, 45, 617620; doi: 10.1016/j.geobios.2012.07.001.
Jiménez-Fuentes, E. \& Martín De Jesús, S. 1989. Quelonios fósiles de la Cuenca de Guadix-Baza (Granada). In: Geología y Paleontología de la Cuenca de Guadix-Baza Trabajos sobre el Neógeno-Cuaternario (eds Alberdi, M. T. \& Bonadonna, F.). Museo Nacional de Ciencias Naturales, CSIC, Madrid, 11, 167-174.

Lacombat, F. 2010. Estudio paleontológico de Stephanorhinus hundsheimensis de Fuente Nueva 3 y Barranco León. In: Ocupaciones Humanas en el Pleistoceno Inferior y Medio de la Cuenca de Guadix-Baza, Memoria Cientifica (eds Toro, I., Martínez-Navarro, B. \& Agustí, J.). Junta de Andalucía, Consejería de Cultura, E.P.G. Arqueología Monográfico, 237-246.

Laplana, C. \& Blain, H.A. 2008. Microvertebrados (Squamata, Lagomorpha, Erinaceomorpha y Rodentia) de Fonelas P-1 (Cuenca de Guadix, Granada). In: Vertebrados del Plioceno Superior Terminal en el Suroeste de Europa: Fonelas P-1 y el Proyecto Fonelas (ed. Arribas, A.). Cuadernos del Museo Geominero, 10, 65-84.

Marín, M. 1987. Equus stenonis granatensis en el Pleistoceno Inferior de Venta Micena (Granada, España). Paleontologia i Evolució, Mem. Esp., 1, 255-282.

Martín-Penela, A. 1983. Presencia del género Macaca en el yacimiento pleistoceno de La Solana del Zamborino (Fonelas, Granada, España). Estudio preliminar. Real Sociedad de Historia Natural, 81, 187-195.

Martín-Penela, A. 1988. Los grandes mamíferos del yacimiento Achelense de la Solana del Zamborino, Fonelas (Granada, España). Antropología y Paleoecología Humana, 5, 29-187.

Martín-Suárez, E. 1988. Sucesiones de micromamíferos en la Depresión de Guadix-Baza (Granada, España). 241 pp. Ph.D. Thesis (unpublished). Univiversidad de Granada, Granada.

Martín-Suárez, E., García-Alix, A., Minwer-Barakat, R., Agustí, J. \& Freudenthal, M. 2012. Filling the gap: First evidence of early Tortonian continental deposits in southern Iberia. Journal of Vertebrate Paleontology, 32, 1421-1428.

Martínez-Navarro, B. 1991. Revisión sistemática y estudio cuantitativo de la fauna de macromamíferos del yacimiento de Venta Micena (Orce, Granada). 264 pp. Ph.D. Thesis, Universidad Autónoma de Barcelona, Barcelona.

Martínez-Navarro, B., Espigares, M.P. \& Ros, S. 2003. Estudio preliminar de las asociaciones de grandes mamíferos de Fuente Nueva-3 y Barranco León-5 (Orce, Granada, España) (informe de las campañas de 19992002). In: El Pleistoceno Inferior de Barranco León y Fuente Nueva 3, Orce (Granada). Memoria Cientifica Campañas 1999-2002 (eds Toro, I., Agustí, J. \& MartínezNavarro, B.). Junta de Andalucía, Consejería de Cultura, E.P.G. Arqueología Monográfico, 115-136.

Martínez-Navarro, B., Palmqvist, P., Madurell, J., RosMontoya, S., Espigares, M.P., Torregrosa, V. \& PérezClaros, J.A. 2010. La fauna de grandes mamíferos de Fuente Nueva 3 y Barranco León 5. Estado de la cuestión. In Ocupaciones Humanas en el Pleistoceno Inferior y Medio de la Cuenca de Guadix-Baza, Memoria Cientifica 
(eds Toro, I., Martínez-Navarro, B. \& Agustí, J.). Junta de Andalucía, Consejería de Cultura, E.P.G. Arqueología Monográfico, 197-236.

Martínez-Navarro, B., Rivals, F., Ros-Montoya, S., Espigares, M.P. \& Palmqvist, P. 2009. Presence of two different Buffaloes at the site of Venta Micena (Orce, Spain). SEQS Annual Meeting "The Quaternary southern Spain: a bridge between Africa and the Alpine domain". Libro de Resúmenes, Orce, 67 p.

Martínez-Navarro, B., Toro, I. \& Agustí, J. 2004. Las asociaciones de grandes mamíferos de Fuente Nueva-3 y Barranco León-5 (Orce, Granada, España): Resultados preliminares. In Miscelánea Homenaje a Emiliano Aguirre, Paleontología (eds Baquedano, E. \& Rubio, S.). Museo Arqueológico Regional, Alcalá de Henares, Madrid, 292-305.

Martínez-Navarro, B., Ros-Montoya, S., Jiménez-Arenas, J.M., Espigares, M.P., Guerra-Merchán, A., GarcíaAguilar, J.M., Rodríguez-Rueda, A., Oms, O., Agustí, J. \& Palmqvist, P. 2015. El yacimiento paleontológico de Baza-1: un nuevo referente del Rusciniense español. XXXI Jornadas de Paleontología. 183-186.

Mazo, A. 1989a. Los hipopótamos del Pleistoceno Medio de Huéscar-1 (Granada). In: Geología y Paleontología de la Cuenca de Guadix-Baza Trabajos sobre el NeógenoCuaternario (eds Alberdi, M.T. \& Bonadonna, F.). Museo Nacional de Ciencias Naturales, CSIC, Madrid, 11, 317-326.

Mazo, A. 1989b. Nuevos restos de Proboscidea (Mammalia) en la cuenca de Guadix-Baza. In: Geología y Paleontología de la Cuenca de Guadix-Baza Trabajos sobre el NeógenoCuaternario (eds Alberdi, M.T. \& Bonadonna, F.). Museo Nacional de Ciencias Naturales, CSIC, Madrid, 11, 225 236.

Mazo, A., Sesé, S., Ruíz Bustos, A. \& Peña, J.A. 1985. Geología y Paleontología de los yacimientos PlioPleistocenos de Huéscar (Depresión de Guadix-Baza, Granada). Estudios Geológicos, 41, 467-493.

Mein, P. 1975. Résultats du groupe de travail des vertébrés: Biozonation du Néogène méditerranéen à partir des mammifères. In: Report on Activity of the RCMNS Working Groups (1971-1975) (ed. Senes, J.). Bratislava, 78-81.

Menéndez, E. 1987. Cérvidos del yacimiento del Pleistoceno inferior de Venta Micena 2, Orce (Granada, España). Paleontologia i Evolucio, Mem. Esp., 1, 129-180.

Minwer-Barakat, R. 2005. Roedores e Insectívoros del Turoliense superior y el Plioceno del Sector Central de la Cuenca de Guadix. 606 pp. Ph.D. Thesis (unpublished). Universidad de Granada.

Minwer-Barakat, R., García-Alix, A., Martín-Suárez, E. \& Freudenthal, M. 2004. Arvicolidae (Rodentia) from the Pliocene of Tollo de Chiclana (Granada, SE Spain). Geobios, 37, 619-629; doi: 10.1016/j.geobios.2004.05.001.

Minwer-Barakat, R., García-Alix, A., Martín-Suárez, E. \& Freudenthal, M. 2005. Muridae (Rodentia) from the Pliocene of Tollo de Chiclana (Granada, Southeastern Spain). Journal of Vertebrate Paleontology, 25, 426-441.
Minwer-Barakat, R., García-Alix, A., Martín-Suárez, E. \& Freudenthal, M. 2007. Blarinoides aliciae sp. nov., a new Soricidae (Mammalia, Lipotyphla) from the Pliocene of Spain. Comptes Rendus Palevol, 6, 281-289; doi: 10.1016/j.crpv.2007.03.001.

Minwer-Barakat, R., García-Alix, A. \& Freudenthal, M. 2008a. Desmaninae (Talpidae, Mammalia) from the Pliocene of Tollo de Chiclana (Guadix Basin, Southern Spain). Considerations on the phylogeny of the genus Archaeodesmana. Geobios, 41, 381-398; doi: 10.1016/j. geobios.2007.08.001.

Minwer-Barakat, R., García-Alix, A., Martín-Suárez, E. \& Freudenthal, M. 2008b. The latest Ruscinian and early Villanyian Arvicolinae from southern Spain re-examined: Biostratigraphical implications. Journal of Vertebrate Paleontology, 28, 841-850.

Minwer-Barakat, R., García-Alix, A., Martín-Suárez, E. \& Freudenthal, M. 2008c. Micromys caesaris, a new murid (Rodentia, Mammalia) from the late Pliocene of the Guadix Basin, Southeastern Spain. Journal of Paleontology, 82, 436-441.

Minwer-Barakat, R., García-Alix, A., Agustí, J., MartínSuárez, E. \& Freudenthal, M. 2009a. The micromammal fauna from Negratín-1 (Guadix Basin, Southern Spain): new evidence of African-Iberian mammal exchanges during the Late Miocene. Journal of Paleontolgy, 83, 854-879.

Minwer-Barakat, R., García-Alix, A., Martín-Suárez, E. \& Freudenthal, M. 2009b. Late Turolian micromammals from Rambla de Chimeneas-3: considerations on the oldest continental faunas from the Guadix Basin (Southern Spain). Neues Jahrbuch für Geologie und Paläontologie Abhandlungen, 251, 95-108; doi: 10.1127/00777749/2009/0251-0095.

Minwer-Barakat, R., García-Alix, A., Martín-Suárez, E. \& Freudenthal, M. 2010. Soricidae (Soricomorpha, Mammalia) from the Pliocene of Tollo de Chiclana (Guadix Basin, Southern Spain). Journal of Vertebrate Paleontology, 30, 535-546.

Minwer-Barakat, R., García-Alix, A., Martín-Suárez, E. \& Freudenthal, M., 2012a. The late Miocene continentalization of the Guadix Basin (southern Spain) reconsidered: a comment on Hüsing et al. (2010). Geobios, 45, 61-615; doi: 10.1016/j.geobios.2012.05.001.

Minwer-Barakat, R., García-Alix, A., Martín-Suárez, E., Freudenthal, M. \& Viseras, C. 2012b. Micromammal biostratigraphy of the Upper Miocene to lowest Pleistocene continental deposits of the Guadix basin, southern Spain. Lethaia, 45, 594-614; doi:10.1111/j.15023931.2012.00324.x.

Moyà-Solà, S. 1987. Los bóvidos (Artiodactyla, Mammalia) del yacimiento del Pleistoceno inferior de Venta Micena (Orce, Granada, España). Paleontologia i Evolució, Mem. Esp., 1, 181-236.

Moyà-Solà, S., Agustí, J. \& Marín, M. 1987. Fuentesnuevas-1: nueva localidad con mamíferos del Plioceno superior de Guadix-Baza (Granada, España). Paleontologia $i$ Evolució, Mem. Esp., 1, 87-93. 
Oms, O., Agustí, J., Gabàs, M. \& Anadón, P. 2000. Lithostratigraphical correlation of micromammal sites and biostratigraphy of the Upper Pliocene to Lower Pleistocene in the Northeast Guadix-Baza Basin, (southern Spain). Journal of Quaternary Science, 15, 43-50; doi: 10.1002/(SICI)1099-1417(200001)15:1<43::AIDJQS475>3.0.CO;2-9.

Piñero, P. \& Agustí, J. 2015. Completando la secuencia bioestratigráfica del Plioceno de la Cuenca de GuadixBaza. XXXI Jornadas de Paleontología. pp. 249-251.

Piñero, P., Agustí, J., Oms, O, Blain, H.A, Laplana, C., RosMontoya, S. \& Martínez-Navarro, B. In press. Rodents from Baza-1 (Guadix-Baza Basin, SE Spain). Filling the gap of the early Pliocene succession in the Betics. Journal of Vertebrate Paleontology.

Pons-Moyà, J. 1987. Los carnívoros (Mammalia) de Venta Micena (Granada, España). Paleontologia i Evolució, Mem. Esp. 1, 109-127.

Ros-Montoya, S. 2010. Los Proboscídeos del Plio-Pleistoceno de las Cuencas de Guadix-Baza y Granada. 403 pp. Ph.D. Thesis (unpublished). Universidad de Granada, Granada.

Ros-Montoya, S., Palombo M.R., Espigares M.P. \& Martínez Navarro, B. 2010. La sucesión de Proboscídeos en el PlioPleistoceno de las cuencas de Guadix-Baza y de Granada (España). In: Ocupaciones Humanas en el Pleistoceno Inferior y Medio de la Cuenca de Guadix-Baza, Memoria Cientifica (eds Toro, I., Martínez-Navarro, B. \& Agustí, J.). Junta de Andalucía, Consejería de Cultura, E.P.G. Arqueología Monográfico., 247-272.

Ros-Montoya, S., Martínez-Navarro, B., Espigares, M.P., Guerra-Merchán, A., García-Aguilar, J.M., Piñero, P., Rodríguez-Rueda, A., Agustí, J., Oms, O. \& Palmqvist, P. In press. A new Ruscinian site in Europe: Baza-1 (Baza Basin, Andalusia, Spain). Comptes Rendus. Paleovol.

Ruíz-Bustos, A. 1976. Estudio sistemático y ecológico sobre las faunas del Pleistoceno medio en las Depresiones granadinas. El yacimiento de Cúllar de Baza I. 309 pp. Ph.D. Thesis (unpublished). Universidad de Granada, Granada.

Ruiz Bustos, A. 1990. Biostratigraphy of the continental Neogene in the Betic Cordilleras. In: Global Events and Neogene Evolution of the Mediterranean (ed. Agustí, J.). Institut Paleontològic M. Crusafont, Sabadell, Barcelona, 301-302.

Ruiz Bustos, A. 1991. Biostratigrafía de los sedimentos neógenos del sector de Galera (Noroeste de la cuenca de Baza, Cordilleras Béticas). I Congreso del Grupo Español del Terciario. Vic, Barcelona, 301-304.

Ruiz Bustos, A., Sesé, C., Dabrio, C.J., Peña, J.A. \& Padial, J. 1984. Geología y fauna de micromamíferos del nuevo yacimiento del Plioceno inferior de Gorafe A (Depresión de Guadix-Baza, Granada). Estudios Geológicos, 40, 231-241.

Sánchez, A. 1989. Huéscar-1 (Granada, España): Avance de la lista de aves y consiguientes conjeturas sobre paleoambiente y paleografía. In: Geología y Paleontología de la Cuenca de Guadix-Baza. Trabajos sobre el NeógenoCuaternario (eds Alberdi, M.T. \& Bonadonna, F.). Museo
Nacional de Ciencias Naturales, CSIC, Madrid, 11, 175184.

Santafé-Llopis, J.V. \& Casanovas-Cladellas, M.L. 1987. Dicerorhinus etruscus brachycephalus (Mammalia, Perissodactyla) de los yacimientos Pleistocénicos de la Cuenca Guadix-Baza (Venta Micena y Huéscar) Granada, España. Paleontologia i Evolució, Mem. Esp., 1, 237-254.

Sesé, C. 1989. Micromamíferos del Mioceno, Plioceno y Pleistoceno de la cuenca de Guadix-Baza (Granada). In: Geología y Paleontología de la Cuenca de Guadix-Baza. Trabajos sobre el Neógeno-Cuaternario (eds Alberdi, M.T. \& Bonadonna, F.). Museo Nacional de Ciencias Naturales, CSIC, Madrid, 11, 185-214.

Soria Mingorance, J.M. \& Ruiz Bustos, A. 1991. Biostratigrafia de los sedimentos continentales del sector septentrional de la cuenca de Guadix, Cordilleras Béticas. Geogaceta, 9, 94-96.

Soria, J.M. \& Ruiz Bustos, A. 1992. Nuevos datos sobre la edad del inicio de la sedimentación continental en la Cuenca de Guadix. Cordillera Bética. Geogaceta, 11, 92-94.

Soria, J.M., Viseras, C. \& Fernández, J. 1998. Late MiocenePleistocene tectonosedimentary evolution and subsidence history of the central Betic Cordillera (Spain): a case study in the Guadix intramontane basin. Geological Magazine, $135,565-574$

Toro, I., Agustí, J. \& Martínez-Navarro, B. 2003. El Pleistoceno Inferior de Barranco León y Fuente Nueva 3, Orce (Granada). Memoria Científica Campañas 19992002. Junta de Andalucía, Consejería de Cultura, E.P.G. Arqueología Monográfico, 17.

Toro, I., Almohalla, M. \& Martín-Suárez, E. 1984. El Paleolítico en Granada. Museo Arqueológico de Granada, $1-67$.

Toro, I., Martínez-Navarro, B. \& Agustí, J. 2010. Ocupaciones Humanas en el Pleistoceno Inferior y Medio de la Cuenca de Guadix-Baza, Memoria Científica. Junta de Andalucía. Consejería de Cultura, E.P.G. Arqueología Monográfico, $582 \mathrm{pp}$.

Toro, I., Martínez-Navarro, B., Agustí, J., Souday, C., Bermúdez de Castro, J.M., Martinón-Torres, M., Fajardo, B., Duval, M., Falguères, C., Oms, O., Parés, J.M., Anadón, P., Julià, R., García-Aguilar, J.M., Moigne, A-M., Espigares, M.P., Ros-Montoya, S. \& Palmqvist, P. 2013. The oldest human fossil in Europe, from Orce (Spain). Journal of Human Evolution, 65, 1-9; doi: 10.1016/j. jhevol.2013.01.012.

Van der Made, J. 1989a. A range-chart for European Suidae and Tayassuidae. Paleontologia i Evolució, 23, 99 - 104.

Van der Made, J. 1989b. Iberian Suoidea. Paleontologia $i$ Evolució, 23, 83.

Van der Made, J. \& Morales, J. 2012. Mitilanotherium inexpectatum (Giraffidae, Mammalia) from Huélago (Lower Pleistocene; Guadix-Baza basin, Granada, Spain)observations on a peculiar biogeographic pattern. Estudios Geológicos, 67, 613-627.

Vera, J.A. 1970. Estudio estratigráfico de la Depresión de Guadix-Baza. Boletin Geológico Minero, 84, 429-462. 
Viseras, C. 1991. Estratigrafía y sedimentología del relleno aluvial de la cuenca de Guadix (Cordilleras Béticas). Ph.D. Thesis (unpublished). Universidad de Granada, Granada. 327 pp.

Viseras, C., Soria, J.M. \& Fernández, J. 2004. Cuencas neógenas postorogénicas de la Cordillera Bética. In: Geología de España (ed. Vera, J.A.). Sociedad Geológica de España-Instituto Geológico y Minero de España, Madrid, 576-581.

Viseras, C., Soria, J.M., Fernández, J. \& García García, F. 2005. The Neogene-Quaternary basins of the Betic Cordillera: an overview. Geophysical Research Abstracts, 7, 11123-11127. 
